\title{
THE DISTRIBUTION OF SPACINGS BETWEEN QUADRATIC RESIDUES
}

\author{
PÄR KURLBERG AND ZEÉV RUDNICK
}

\begin{abstract}
We study the distribution of spacings between squares modulo $q$, where $q$ is square-free and highly composite, in the limit as the number of prime factors of $q$ goes to infinity. We show that all correlation functions are Poissonian, which among other things, implies that the spacings between nearest neighbors, normalized to have unit mean, have an exponential distribution.
\end{abstract}

Date: Dec 14, 1998.

Supported in part by a grant from the Israel Science Foundation. In addition, the first author was partially supported by the EC TMR network "Algebraic Lie Representations", EC-contract no ERB FMRX-CT97-0100. 


\section{INTRODUCTION}

Our goal in this paper is to study the distribution of spacings (or gaps) between squares in $\mathbf{Z} / q \mathbf{Z}$, as $q \rightarrow \infty$. In the case that $q$ is prime, a theorem of Davenport [3, 1, 11, 18] shows that the probability of two consecutive quadratic residues modulo a prime $q$ being spaced $h$ units apart is $2^{-h}$, as $q \rightarrow \infty$. For our purposes, we may interpret this result as saying that when we normalize the spacings to have unit mean, then the distribution of spacing as $q \rightarrow \infty$ along primes is given by

$$
P(s)=\sum_{h=1}^{\infty} 2^{-h} \delta\left(s-\frac{h}{2}\right)
$$

that is, a sum of point masses at half-integers with exponentially decreasing weights.

In this paper we study the spacing distribution of squares modulo $q$ when $q$ is square-free and highly composite, that is the limiting distribution of spacings between the squares modulo $q$ as the number of prime divisors, $\omega(q)$, tends to infinity. For odd square-free $q$ the number $N_{q}$ of squares modulo $q$ equals

$$
N_{q}=\prod_{p \mid q} \frac{p+1}{2}
$$

This is because if $p$ is an odd prime, the number of squares modulo $p$ is $(p+1) / 2$ and for $q$ square-free, $x$ is a square modulo $q$ if and only if $x$ is a square modulo $p$ for all primes $p$ dividing $q$. Thus for odd $q$, the mean spacing $s_{q}=q / N$ equals

$$
s_{q}=\frac{2^{\omega(q)}}{\prod_{p \mid q}(1+1 / p)}=\frac{2^{\omega(q)}}{\sigma_{-1}(q)}
$$

For $q=2 q^{\prime}$ even and square-free, it is easily seen that $s_{q}=s_{q^{\prime}}$. It follows that $s_{q} \rightarrow \infty$ as $\omega(q) \rightarrow \infty$, unlike the case of prime $q$ where the mean spacing is essentially constant. Thus, unlike in the prime case where the level spacing distribution was forced to be supported on a lattice, in the highly composite case there is an a-priori chance of getting a continuous distribution.

A relevant statistical model for the distribution of spacings is given by looking at random points in the unit interval $\mathbf{R} / \mathbf{Z}$. For independent, uniformly distributed numbers in $\mathbf{R} / \mathbf{Z}$, the spacing statistics are said to be Poissonian. The distribution $P(s)$ of spacings between consecutive points will be that of a Poisson arrival process, i.e. $P(s)=e^{-s}$ (see [6]). Moreover, the joint distribution of $k$ consecutive spacings is the product of $k$ independent exponential random variables. 
It is well known [15] that the spacing statistics of the superposition of several independent spectra converges to the Poisson case - the spacings statistics of uncorrelated levels. Thus the heuristic that "primes are independent" together with Davenport's result indicates that the spacing statistics of the squares modulo $q$ should in the limit as $\omega(q) \rightarrow \infty$ be Poissonian, i.e., that in some sense squares modulo $q$ behaves as random numbers. It is our purpose to confirm this expectation.

In order to study the level spacings, we proceed by studying the $r$ - level correlation functions. These measure clustering properties of a sequence in $\mathbf{R} / \mathbf{Z}$ on a scale of the mean spacing. Their definition and their application to computing various local spacings statistics are recalled in appendix A. In our case, these turn out to be given by by the following: For $r \geq 2$ and a bounded convex set $\mathcal{C} \subset \mathbf{R}^{r-1}$, let

$R_{r}(\mathcal{C}, q)=\frac{1}{N_{q}} \#\left\{x_{i}\right.$ distinct squares $\left.\bmod q:\left(x_{1}-x_{2}, \ldots x_{r-1}-x_{r}\right) \in s \mathcal{C}\right\}$.

This is immediately transformed into

$$
R_{r}(\mathcal{C}, q)=\frac{1}{N_{q}} \sum_{h \in s \mathcal{C} \cap \mathbf{Z}^{r-1}} N(h, q)
$$

where $N(h, q)$ is the number of solutions of the system of congruences $y_{i+1}-y_{i}=h_{i} \bmod q$ with $y_{1}, y_{2}, \ldots y_{r}$ squares modulo $q$ and $h=\left(h_{1}, \ldots h_{r-1}\right) \in \mathbf{Z}^{r-1}$.

To compute the correlations for distinct $x_{i}$ we consider only sets $\mathcal{C}$ which a-priori only contain vectors $\left(x_{i}-x_{i+1}\right)$ with distinct coordinates. To do this, we define "roots" $\sigma_{i j}$ on $\mathbf{R}^{r-1}$ for $i<j$ by $\sigma_{i j}(h)=\sum_{k=i}^{j-1} h_{k}$. The hyper-planes $\left\{\sigma_{i j}=0\right\} \subset \mathbf{R}^{r-1}$ are called "walls", and $\left(x_{i}-x_{i+1}\right)$ does not lie in any of the walls if and only if all coordinates $x_{i}$ are distinct.

Our main result shows that if $\mathcal{C}$ does not intersect any wall then $R_{r}(\mathcal{C}, q) \rightarrow \operatorname{vol}(\mathcal{C})$ for any sequence of square-free $q$ with $\omega(q) \rightarrow \infty$ :

Theorem 1. Let $q$ be square-free, $r \geq 2$ and $\mathcal{C} \subset \mathbf{R}^{r-1}$ a bounded convex set which does not intersect any of the walls. Then the $r$-level correlation function satisfies

$$
R_{r}(\mathcal{C}, q)=\operatorname{vol}(\mathcal{C})+O\left(s^{-1 / 2+\epsilon}\right) \quad \text { as } s \rightarrow \infty
$$

for all $\epsilon>0$, where $s$ is the mean spacing.

This theorem implies that all spacing statistics are Poissonian (see Appendix A). For instance, if we denote by $s_{1}, \ldots, s_{N-1}$ the normalized differences between neighboring squares, then we have 
Theorem 2. For q square-free, the limiting level spacing distribution of the squares modulo $q$ is given by $P(t)=\exp (-t)$ as $\omega(q) \rightarrow \infty$. Moreover, under the same condition, for any $k \geq 1$ the limiting joint distribution of $\left(s_{n}, s_{n+1}, \ldots, s_{n+k}\right)$ is a product $\prod_{i=0}^{k} \exp \left(-t_{i}\right)$ of $k+1$ independent exponential variables.

There are only a few known cases where the complete spacing distribution can be proved to be Poissonian as in our case. A notable example is Hooley's results [7, 8, 9, 10] that the spacings between elements co-prime to $q$ are Poissonian as the mean spacing $q / \phi(q) \rightarrow \infty$. A much more recent result is due to Cobeli and Zaharescu [2] who show that the spacings between primitive roots modulo a prime $p$ are Poissonian provided the mean spacing $p / \phi(p-1) \rightarrow \infty$.

The results of this paper are related to work on the level spacing distribution of the fractional parts $\left\{\alpha n^{2}\right\}$ ( $\alpha$ irrational) by Rudnick, Sarnak and Zaharescu [16, 17]. In particular, in [17] an attempt to study that problem is made by replacing $\alpha$ with a rational approximation $b / q$, and this leads to study the spacings of the sequence $b n^{2}$ $\bmod q, 1 \leq n \leq N$ for $N$ a small power of $q$. The available sites are exactly the set of squares modulo $q$, and hence our interest in the problem.

In [17], it is shown that in order for all the correlation functions of the sequence $\left\{\alpha n^{2}\right\}$ to have Poisson behavior, it is necessary to assume that the rational approximants $b / q$ have denominator $q$ which is close to square-free. Hence our interest in the square-free case. For arbitrary $q$ it is still true that all correlations are Poissonian, but there are significant technical complications to overcome in proving this, see 13 .

We believe that the methods developed in this paper should be useful in studying similar problems, for instance the spacing distribution of cubes modulo $q$, as the number of prime factors of $q$ that are congruent to 1 modulo 3 tends to infinity. (The condition modulo 3 is necessary in order for the mean spacing to go to infinity.)

Contents of the paper: We begin with a section sketching the argument for Theorem 11 in the case of the pair correlation function. This section can be used as a guide to the rest of the paper.

In section 3 we first reduce the problem to the case that $q$ is odd. Then in section 4 we analyze the behavior of $N(h, p)$ where $p$ is prime. Squares that are distinct modulo $q$ are not necessarily distinct modulo $p$; we denote by $r_{\text {eff }}(h)$ the number of squares that remains distinct after reduction modulo $p$. Using an inclusion-exclusion argument we 
write $r_{e f f}(h)$ as a linear combination of characteristic functions of certain hyper-planes over $\mathbf{Z} / p \mathbf{Z}$. Next, in section 5 we use the multiplicative properties of the counting functions $N(h, q)$ to derive an expression for $R_{r}(\mathcal{C}, q)$ as a sum over divisors $c$ of $q$ and lattices $L$ arising from intersections of hyper-planes modulo $p$ for different $p$ 's (proposition 6).

In section 6 we show that the main term of the sum consists of those terms for which the product of $c$ and the discriminant of $L$ are small with respect to $s$, and an error term corresponding to terms where the product is large. In section 7 we evaluate the main term and show that it gives us exactly $\operatorname{vol}(\mathcal{C})$, thus giving us our main result.

In appendix A we explain how to use Theorem 1 to derive results such as Theorem 2, that the level spacings are Poissonian as well. Appendix B explains some background on counting lattice points in convex sets. In appendix $\mathrm{Q}$ we estimate the number of divisors of $q$ that are smaller than a fixed power of the mean spacing $s$. 


\section{THE PAIR CORRELATION - A SKETCH}

In order to explain the proof of our main theorem 1, we give an overview of the argument in the special case of the pair correlation function.

Let $q$ be an odd, square-free number with $\omega(q)$ prime factors, and $I$ an interval, not containing the origin. Define as in the introduction the pair correlation function

$$
R_{2}(I, q)=\frac{1}{N} \sum_{h \in s I \cap \mathbf{Z}} N(h, q)
$$

where $N$ is the number of squares modulo $q, s=q / N=2^{\omega(q)} / \sigma_{-1}(q)$ is their mean spacing, $\sigma_{-1}(q)=\prod_{p \mid q}\left(1+\frac{1}{p}\right)$, and $N(h, q)$ is the number of solutions in squares modulo $q$ of the equation

$$
y_{1}-y_{2}=h \quad \bmod q
$$

We will sketch a proof that $R_{2}(I, q) \rightarrow|I|$ as $\omega(q) \rightarrow \infty(|I|$ being the length of the interval). In fact we have the more precise result

Theorem 3. For $q$ odd, square-free we have for all $\epsilon>0$

$$
R_{2}(I, q)=|I|+O\left(s^{-1+\epsilon}\right)
$$

Here are the main steps in the argument:

\section{Step 1:}

By the Chinese Remainder Theorem, $N(h, q)=\prod_{p \mid q} N(h, p)$ is a product over primes dividing $q$. By elementary considerations, one sees that

$$
N(h, p)=\frac{p+a(h, p)}{4} \Delta(h, p)
$$

with $a(h, p)=O(1)$ and

$$
\Delta(h, p)=1+\delta(h, p), \quad \delta(h, p)= \begin{cases}0 & p \not h \\ 1 & p \mid h\end{cases}
$$

From this we see that

$$
N(h, q)=\frac{q \Delta(h, q)}{4^{\omega(q)}} \sum_{c \mid q} \frac{a(h, c)}{c}
$$

with $a(h, c):=\prod_{p \mid c} a(h, p) \ll c^{\epsilon}$ and $\Delta(h, q)=\prod_{p \mid q} \Delta(h, p)$.

\section{Step 2:}

We decompose $\Delta(h, q)=\Delta(h, c) \Delta\left(h, \frac{q}{c}\right)$ and rewrite $\Delta\left(h, \frac{q}{c}\right)$ as

$$
\Delta\left(h, \frac{q}{c}\right)=\prod_{p \mid \frac{q}{c}}(1+\delta(h, p))=\sum_{g \mid \frac{q}{c}} \delta(h, g)
$$


with

$$
\delta(h, g)= \begin{cases}0 & g \not h \\ 1 & g \mid h\end{cases}
$$

Substituting this into the expression (2.2) for $N(h, q)$ and inserting the result into the formula for $R_{2}(I, q)$, we get

$$
R_{2}(I, q)=\frac{1}{\sigma_{-1}(q) 2^{\omega(q)}} \sum_{c \mid q} \frac{1}{c} \sum_{g \mid \frac{q}{c}} \sum_{h \in s I \cap g \mathbf{Z}} a(h, c) \Delta(h, c)
$$

\section{Step 3:}

We partition the sum into two parts, one over the pairs $g, c$ with $g c<$ $s$ and the leftover part over pairs with $g c \geq s$. We will show this leftover part is negligible (in fact $O\left(s^{-1+\epsilon}\right)$ ): We first use $a(h, c) \Delta(h, c) \ll c^{\epsilon}$ and the fact that in order for the inner sum over $h$ to be nonempty, we need $g \ll s$ (recall that $I$ does not contain the origin!) to get that the sum over pairs with $c g>s$ is bounded by

$$
\begin{aligned}
s^{-1+\epsilon} \sum_{c \mid q} c^{-1+\epsilon} \sum_{\substack{g \mid \frac{q}{c} \\
g \ll s \\
c g>s}} \#(s I \cap g \mathbf{Z}) & \ll s^{-1+\epsilon} \sum_{c \mid q} c^{-1+\epsilon} \sum_{\substack{g \mid \frac{q}{g} \\
g \ll s \\
c g>s}} \frac{s}{g} \\
& \ll s^{\epsilon} \sum_{\substack{d \mid q \\
d>s}} d^{-1+\epsilon} \sum_{\substack{g \mid d \\
g \ll s}} 1
\end{aligned}
$$

Now we use Lemma 18 which shows that the number of divisors $g<s$ of $q$ is a most $O\left(s^{\epsilon}\right)$ and Lemma 19 to bound the above by

$$
s^{\epsilon} \sum_{\substack{d \mid q \\ d>s}} d^{-1+\epsilon} \ll s^{-1+\epsilon}
$$

as promised.

\section{Step 4:}

For each pair of $c, g$ with $c g<s$, we first treat the inner sum over $h \in s I \cap g \mathbf{Z}$. We break it up into sums over $\frac{s|I|}{g c}+O(1)$ subintervals $[y, y+c g) \cap g \mathbf{Z}$ plus a leftover term of size at most $c^{1+\epsilon}$. For each subinterval, we use periodicity of $a(h, c) \Delta(h, c)$ under $h \mapsto h+c$ to find

$$
\sum_{h \in[y, y+c g) \cap g \mathbf{Z}} a(h, c) \Delta(h, c)=\sum_{h_{1}=1}^{c} a\left(g h_{1}, c\right) \Delta\left(g h_{1}, c\right)
$$

Because $q$ is square-free, and $g$ divides $q / c$, we have that $g, c$ are coprime. Therefore we can change variables $h=g h_{1}$ to get that this 
last sum equals

$$
\sum_{h} a(h, c) \Delta(h, c)=\prod_{p \mid c} \sum_{h \bmod p} a(h, p) \Delta(h, p)
$$

We evaluate the sum $\sum_{h \bmod p} a(h, p) \Delta(h, p)$ by noting that summing (2.1) over $h \bmod p$, the sum of the LHS is simply the number of all pairs of squares modulo $p$, namely $(p+1)^{2} / 4$. This gives

$$
\sum_{h} a(h, p) \Delta(h, p)=p+1
$$

Thus the inner sum over $h \in s I \cap g \mathbf{Z}$ equals

$$
\begin{aligned}
\sum_{h \in s I \cap g \mathbf{Z}} a(h, c) \Delta(h, c) & =\left(\frac{s|I|}{g c}+O(1)\right) \prod_{p \mid c}(p+1)+O\left(c^{1+\epsilon}\right) \\
& =\frac{s|I|}{g} \sigma_{-1}(c)+O\left(c^{1+\epsilon}\right)
\end{aligned}
$$

\section{Step 5:}

Inserting this into the expression (2.3) for $R_{2}(I, q)$ gives

$$
R_{2}(I, q)=\frac{1}{2^{\omega(q)} \sigma_{-1}(q)} \sum_{c \mid q} \frac{1}{c} \sum_{g \mid \frac{q}{c}: g c<s} \frac{s|I|}{g} \sigma_{-1}(c)+O\left(s^{-1+\epsilon}\right)
$$

Now we extend the sum to all pairs $g, c$, to find that up to an error of $O\left(s^{-1+\epsilon}\right)$ we have

$$
\begin{aligned}
R_{2}(I, q) & \sim|I| \frac{1}{\sigma_{-1}(q)^{2}} \sum_{c \mid q} \frac{\sigma_{-1}(c)}{c} \sum_{g \mid \frac{q}{c}} \frac{1}{g} \\
& =|I| \frac{1}{\sigma_{-1}(q)^{2}} \sum_{c \mid q} \frac{\sigma_{-1}(c)}{c} \sigma_{-1}\left(\frac{q}{c}\right) \\
& =|I| \frac{1}{\sigma_{-1}(q)} \sum_{c \mid q} \frac{1}{c}=|I|
\end{aligned}
$$

which is what we need to prove our theorem

In the following sections, we will repeat these steps with full details for the higher correlation functions, where several technical complications arise. 


\section{REDUCTION TO ODD $q$}

We first show that in Theorem 1 it suffices to consider only the case of $q$ odd: Suppose that $q=2 q^{\prime}$ with $q^{\prime}$ odd and square-free. We recall that

$$
R_{r}(\mathcal{C}, q)=\frac{1}{N} \sum_{h \in s \mathcal{C} \cap \mathbf{Z}^{r-1}} N(h, q)
$$

where $N(h, q)$ is the number of solutions of the system $y_{i+1}-y_{i}=$ $h_{i}$ where $y_{1}, y_{2}, \ldots y_{r}$ are squares modulo $q$ and $h=\left(h_{1}, \ldots h_{r-1}\right) \in$ $(\mathbf{Z} / q \mathbf{Z})^{r-1}$.

By the Chinese Remainder Theorem, the number $N_{q}$ of squares modulo $q$ is the product

$$
N_{q}=N_{2} N_{q^{\prime}}=2 N_{q^{\prime}}
$$

Therefore the mean spacing $s_{q}:=q / N_{q}$ is given by

$$
s_{q}=\frac{2 q^{\prime}}{2 N_{q^{\prime}}}=\frac{q^{\prime}}{N_{q^{\prime}}}=s_{q^{\prime}}
$$

Moreover, again by the Chinese Remainder Theorem,

$$
N(h, q)=N(h, 2) N\left(h, q^{\prime}\right)
$$

and since all residues modulo 2 are squares, we have $N(h, 2)=2$. Thus we find

$$
\frac{N(h, q)}{N_{q}}=\frac{2 N\left(h, q^{\prime}\right)}{2 N_{q^{\prime}}}=\frac{N\left(h, q^{\prime}\right)}{N_{q^{\prime}}}
$$

Inserting (3.2), (3.3) into (3.1), we find that

$$
R_{r}(\mathcal{C}, q)=R_{r}\left(\mathcal{C}, q^{\prime}\right)
$$

This shows that it suffices to prove Theorem 1 f for $q$ odd, which we assume is the case in the sequel. 


\section{THE PRIME CASE}

Let $p>2$ be a prime. For $h=\left(h_{1}, \ldots h_{r-1}\right) \in(\mathbf{Z} / p \mathbf{Z})^{r-1}$, we define $N_{r}(h, p)$ to be the number of solutions in squares $y_{i} \bmod p$ (including $\left.y_{i}=0\right)$ of the system

$$
y_{i}-y_{i+1}=h_{i} \quad \bmod p, \quad 1 \leq i \leq r-1
$$

This number depends crucially on the number of distinct $y_{j}$. For each $h=\left(h_{1}, \ldots, h_{r-1}\right)$, we define $r_{e f f}(h)$ to be the number of distinct $y_{j}$ (not necessarily squares) satisfying the system (4.1). Since the solutions of the homogeneous system $y_{i}-y_{i+1}=0 \bmod p$ are spanned by $(1, \ldots, 1), r_{e f f}(h)$ is well-defined (independent of the particular solution $y$ of (4.1)).

We define roots $\sigma_{i j}(h), 1 \leq i<j \leq r$ by

$$
\sigma_{i j}(h)=\sum_{k=i}^{j-1} h_{k}
$$

so that $\sigma_{i, i+1}(h)=h_{i}, \sigma_{i j}=\sum_{k=i}^{j-1} \sigma_{k, k+1}$. The solutions of (4.1) are all distinct of and only if $\sigma_{i j}(h) \neq 0$, for all $i<j$, since

$$
y_{i}-y_{j}=\sum_{k=i}^{j-1} y_{k}-y_{k+1}=\sum_{k=i}^{j-1} h_{k}=\sigma_{i j}(h)
$$

Proposition 4. Let $r_{\text {eff }}(h)$ be the number of distinct $y_{i}$ in a solution of (4.1). Then

$$
N_{r}(h, p)=\frac{p+a(h, p)}{2^{r_{e f f}}}
$$

with $a(h, p) \ll_{r} p^{1 / 2}$.

Proof. The case $r_{\text {eff }}(h)=1$ is precisely when $h=0$ and all $y_{i}$ are equal: $y_{1}=y_{2}=\cdots=y_{r}$. In this case the number of solutions is the number of squares modulo $p$, namely $(p+1) / 2$, which is of the desired form. We thus assume from now that $r_{\text {eff }}(h)>1$.

We first reduce the system (4.1) to a system of $r_{\text {eff }}-1$ equations in $r_{\text {eff }}$ variables: If $r_{e f f}(h)$ is the number of distinct $y_{i}$ in a solution of (4.1) (independent of $y$ !), then we can eliminate some of the equations. Renumber the variables so that $y_{1}, \ldots y_{r_{e f f}}$ are the distinct coordinates of a solution, and for all $j \geq 1, y_{r_{e f f}+j}$ equals one of these, then the system (4.1) is equivalent to the reduced system

$$
y_{i}-y_{i+1}=h_{i}^{\prime} \quad \bmod p, \quad 1 \leq i \leq r_{e f f}-1
$$


(where the $h_{i}^{\prime}$ are renumbered $h_{j}$ to give that the first $r_{\text {eff }}$ coordinates are distinct). So we need to find the number of solution of the reduced system (4.4).

We first eliminate those solutions where at least one of the $y_{j}$ is zero. In this case, since the system (4.4) (considered as a linear system) has rank $r_{e f f}-1$ in $r_{e f f}$ variables, specifying any one of the variables determines all the others, hence the number of solutions with some coordinate zero is at most $r_{\text {eff }}$. Thus we need only count solutions where all coordinates $y_{i}$ are nonzero.

To every such solution in squares $y_{i} \neq 0 \bmod p$, write $y_{i}=x_{i}^{2}$ $\bmod p$ with $x_{i} \neq 0 \bmod p$. There are precisely two such solutions, namely $\pm x_{i} \bmod p$. Thus the number of possible $x_{i}$ corresponding to a given solution $y$ of (4.4) is precisely $2^{r_{e f f}}$, and the number of nonzero solutions of the reduced system (4.4) with $y_{i}$ squares modulo $p$ is exactly $1 / 2^{r_{\text {eff }}}$ times the number of solutions of the system

$$
x_{i}^{2}-x_{i+1}^{2}=h_{i}^{\prime} \quad \bmod p, \quad 1 \leq i \leq r_{e f f}-1
$$

with $x_{i} \neq 0 \bmod p$. By adding back at most $r$ solutions we can remove the condition $x_{i} \neq 0$, and then we find that

$$
N_{r}(h, p)=\frac{1}{2^{r_{e f f}}} n\left(h^{\prime}, p\right)+O_{r}(1)
$$

where $n\left(h^{\prime}, p\right)$ is the number of solutions of

$$
x_{i}^{2}-x_{i+1}^{2}=h_{i}^{\prime} \quad \bmod p \quad 1 \leq i \leq r_{e f f}(h)-1
$$

This is just the number of solutions $\left(t, x_{1}, \ldots, x_{r_{e f f}}\right)$ of the system

$$
x_{1}^{2}=t-b_{1}, \quad x_{2}^{2}=t-b_{2}, \ldots, x_{r_{e f f}}^{2}=t-b_{r_{e f f}}
$$

with $b_{1}=0, b_{2}=h_{1}^{\prime}, b_{3}=h_{1}^{\prime}+h_{2}^{\prime}, \ldots, b_{r_{e f f}(h)}=h_{1}^{\prime}+h_{2}^{\prime}+\cdots+$ $h_{r_{e f f}-1}^{\prime}$ and in general $b_{k}=\sigma_{1 k}\left(h^{\prime}\right)$. Note that the $b_{i}$ are distinct this is equivalent to the requirement that the solutions of the reduced system (4.4) be distinct. One can now use the "Riemann Hypothesis for curves" 21] (see Schmidt's book 19], Chapter II, Theorem 5A and Corollary 5B for the case $b_{1}=-1, b_{2}=-2, \ldots b_{r}=-r$ ), to find

$$
\left|n\left(h^{\prime}, p\right)-p\right| \ll r_{e f f} 2^{r_{e f f}} \sqrt{p}
$$

In addition, $\left|N(h, p)-n\left(h^{\prime}, p\right) / 2^{r_{e f f}}\right| \leq r$ and so

$$
N(h, p)=\frac{p+a(h, p)}{2^{r_{e f f}}}
$$

with

$$
a(h, p) \ll 2^{r_{e f f}}\left(r_{e f f} \sqrt{p}+r\right) \ll_{r} \sqrt{p}
$$

This proves Proposition 1 . 
4.1. A formula for $r_{e f f}(h)$. Our next order of business is to give a formula for $r_{e f f}(h)$. We begin with some combinatorial background: A set partition of the set $\{1,2, \ldots, r\}$ is a collection of disjoint subsets $\underline{F}=\left[F_{1}, \ldots, F_{t}\right], F_{i} \subseteq\{1,2, \ldots, r\}$, whose union is all of $\{1,2, \ldots, r\}$. We set $|\underline{F}|=t$, the number of subsets in $\underline{F}$.

To each set partition $\underline{F}$, we associate a subset $V_{F}$ of affine $r$-space $V=\mathbf{A}^{r}$ by setting

$$
V_{\underline{F}}=\left\{s \in \mathbf{A}^{r}: s_{i}=s_{j} \text { if } i, j \text { are in some } F_{k}\right\}
$$

Correspondingly, in $H=\mathbf{A}^{r-1}$ we have a subspace

$$
H_{\underline{F}}=\left\{h \in \mathbf{A}^{r-1}: \sigma_{i j}(h)=0 \text { if } i, j \text { are in some } F_{k}\right\}
$$

Under the map $\pi: V \rightarrow H$ taking $s=\left(s_{i}\right) \mapsto\left(s_{i}-s_{i+1}\right)$, we have $V_{\underline{F}}=\pi^{-1} H_{\underline{F}}$.

There is a partial ordering on the collection of all set-partitions of $\{1, \ldots, r\}$ with $\underline{F} \preceq \underline{G}$ if and only if every $F_{i}$ is contained in some $G_{j}$.

For example, $\underline{O}=[\{1,2, \ldots, r\}]$ is the maximal element of this partial ordering, with $|\underline{O}|=1$ and $H_{\underline{O}}=(0)$. The minimal element is $\underline{r}=[\{1\},\{2\}, \ldots,\{r\}]$ with $|\underline{r}|=r$ and $H_{\underline{r}}=\mathbf{A}^{r-1}$.

The partial ordering on set-partitions is inclusion-reversing on subspaces: $\underline{F} \preceq \underline{G} \Leftrightarrow V_{\underline{F}} \supseteq V_{\underline{G}} \Leftrightarrow H_{\underline{F}} \supseteq H_{\underline{G}}$.

The regular part of $V_{\underline{F}}$ is

$$
V_{\underline{F}}^{\times}=\left\{s \in V_{\underline{F}}: s_{i} \neq s_{j} \text { if } i, j \text { are not in some } F_{k}\right\}
$$

and likewise we define $H_{\underline{F}}^{\times}$. Then $H_{\underline{O}}^{\times}=H_{\underline{O}}=(0)$, and every $h$ belongs to a unique $H_{\underline{F}}^{\times}$for some $\underline{F}$. We thus have

$$
H=\coprod_{\underline{F}} H_{\underline{F}}^{\times}
$$

and likewise

$$
H_{\underline{F}}=\coprod_{\underline{F} \preceq \underline{G}} H_{\underline{G}}^{\times}
$$

We can now give a formula for $r_{\text {eff }}(h)$ :

$$
r_{e f f}(h)=\operatorname{dim} V_{\underline{F}}=\operatorname{dim} H_{\underline{F}}+1=|\underline{F}|
$$

where $\underline{F}$ is the unique set-partition such that $h \in H_{F}^{\times}$.

We can write this as follows: Define

$$
\delta_{\underline{F}}(h)=\left\{\begin{array}{ll}
1 & h \in H_{\underline{F}} \\
0 & \text { otherwise }
\end{array}, \quad \delta_{\underline{F}}^{\times}(h)= \begin{cases}1 & h \in H_{\underline{F}}^{\times} \\
0 & \text { otherwise }\end{cases}\right.
$$


Then

$$
r_{e f f}(h)=\sum_{\underline{F}} \operatorname{dim}\left(V_{\underline{F}}\right) \delta_{\underline{F}}^{\times}(h)
$$

Similarly

$$
\Delta(h, p):=2^{r-r_{e f f}(h)}=\sum_{\underline{F}} 2^{\operatorname{codim}\left(V_{\underline{F}}\right)} \delta_{\underline{F}}^{\times}(h)
$$

It will be convenient to express this in terms of the characteristic function $\delta_{\underline{F}}$ of the subspaces $H_{\underline{F}}$. For this we use Möbius inversion. Since the collection of all set-partitions of $\{1, \ldots, r\}$ is a partially-ordered set, it has a Möbius function $\mu(\underline{F}, \underline{G})$ which is the unique function so that for any functions $\psi, \phi$ on set-partitions satisfying

$$
\phi(\underline{F})=\sum_{\underline{F} \preceq \underline{G}} \psi(\underline{G})
$$

we have

$$
\psi(\underline{F})=\sum_{\underline{F} \preceq \underline{G}} \mu(\underline{F}, \underline{G}) \phi(\underline{G})
$$

An explicit form of $\mu(\underline{F}, \underline{G})$ can be found in [14], $\S 25$. We will not have any use for it.

In our case, clearly we have $H_{\underline{F}}=\coprod_{\underline{F} \preceq \underline{G}} H_{\underline{G}}^{\times}$so that

$$
\delta_{\underline{F}}=\sum_{\underline{F} \preceq \underline{G}} \delta_{\underline{G}}^{\times}
$$

Thus we have

$$
\delta_{\underline{F}}^{\times}=\sum_{\underline{F} \preceq \underline{G}} \mu(\underline{F}, \underline{G}) \delta_{\underline{G}}
$$

This gives us the formula for $\Delta(h, p)=2^{r-r_{e f f}(h)}$ : From (4.14) and (4.18) we find

$$
\Delta(h, p)=\sum_{\underline{G}} \lambda(\underline{G}) \delta_{\underline{G}}(h)
$$

with

$$
\lambda(\underline{G})=\sum_{\underline{F} \preceq \underline{G}} \mu(\underline{F}, \underline{G}) 2^{\operatorname{codim} V_{\underline{F}}}
$$

For use in Section 7, we need to know the sum of the product of $\Delta(h, p)$ with the error term $a(h, p)$ in (4.3) over all vectors $h$ : 


\section{Lemma 5.}

$$
\sum_{h} a(h, p) \Delta(h, p)=(p+1)^{r}-p^{r} \sum_{\underline{G}} \lambda(\underline{G}) p^{-\operatorname{codim} H_{\underline{G}}}
$$

Proof. We have by definition

$$
N(h, p)=\frac{p+a(h, p)}{2^{r}} \Delta(h, p)
$$

so that

$$
a(h, p) \Delta(h, p)=2^{r} N(h, p)-p \Delta(h, p)
$$

Now sum over all $h \bmod p$ : The sum of $N(h, p)$ is just the total number of $r$-tuples of squares modulo $p$, namely $\left(\frac{p+1}{2}\right)^{r}$. To sum $\Delta(h, p)$ over $h$, we use (4.19): Since the sum over all $h$ of $\delta_{\underline{G}}(h)$ is just the number of vectors in the subspace $H_{\underline{G}}$, namely $p^{\operatorname{dim} H_{\underline{G}}}=p^{r-1-\operatorname{codim} H_{\underline{G}}}$, we find

$$
\begin{aligned}
\sum_{h \bmod p} a(h, p) \Delta(h, p) & =(p+1)^{r}-p \sum_{h} \Delta(h, p) \\
& =(p+1)^{r}-p^{r} \sum_{\underline{G}} \lambda(\underline{G}) p^{-\operatorname{codim} H_{\underline{G}}}
\end{aligned}
$$

as required. 


\section{A FORMUla FOR $R_{r}(\mathcal{C}, q)$}

In order to prove Theorem 1, we give an expression (5.2) for the $r$ level correlation $R_{r}(\mathcal{C}, q)$ which involves summing over the intersection of the dilated set $s \mathcal{C}$ with various lattices.

Recall that for each set-partition $\underline{G}$ of $\{1, \ldots, r\}$ we associated a subspace $H_{\underline{G}} \subseteq(\mathbf{Z} / p \mathbf{Z})^{r-1}$. Now given a divisor $d \bmod q$, let $\mathcal{G}=$ $\otimes_{p \mid d} \underline{G}^{(p)}$, be a tuple of such set-partitions, one for each prime $p$ dividing $d$ (recall that $q$, hence $d$, is square-free). Let $L(\mathcal{G}) \subset \mathbf{Z}^{r-1}$ be the preimage of $\prod_{p \mid d} H_{\underline{G}^{(p)}}$ under the reduction map $\mathbf{Z}^{r-1} \rightarrow \prod_{p \mid d}(\mathbf{Z} / p \mathbf{Z})^{r-1} \simeq$ $(\mathbf{Z} / d \mathbf{Z})^{r-1} . L(\mathcal{G})$ is a lattice, whose discriminant (that is, the index in $\left.\mathbf{Z}^{r-1}\right)$ is

$$
\operatorname{disc}(\mathcal{G})=\prod_{p \mid d} p^{\operatorname{codim}\left(H_{\underline{G}}(p)\right)}
$$

The support $\operatorname{supp}(\mathcal{G})$ of $L(\mathcal{G})$ is the product of all primes $p$ for which $H_{\underline{G}^{(p)}} \neq(\mathbf{Z} / p \mathbf{Z})^{r-1}$ :

$$
\operatorname{supp}(\mathcal{G})=\prod_{p: \underline{G}^{(p)} \neq[\{1\}, \ldots,\{r-1\}]} p
$$

Since $\operatorname{codim}\left(H_{\underline{G}^{(p)}}\right) \leq r-1$, we get

$$
\operatorname{supp}(\mathcal{G})|\operatorname{disc}(\mathcal{G})| \operatorname{supp}(\mathcal{G})^{r-1}
$$

We set

$$
\lambda(\mathcal{G})=\prod_{p \mid d} \lambda\left(\underline{G}^{(p)}\right)
$$

where $\lambda(\underline{G})$ is given by (4.20). We also set for a divisor $c \mid q$

$$
a(h, c):=\prod_{p \mid c} a(h, p), \quad \Delta(h, c):=\prod_{p \mid c} \Delta(h, p)
$$

Note that by Proposition 1

$$
a(h, c) \ll c^{1 / 2+\epsilon}, \quad \Delta(h, c) \ll c^{\epsilon}
$$

for all $\epsilon>0$.

Our formula for $R_{r}(\mathcal{C}, q)$ is

Proposition 6. The r-level correlation function is given by

$$
R_{r}(\mathcal{C}, q)=\frac{s}{2^{r \omega(q)}} \sum_{c \mid q} \frac{1}{c} \sum_{\operatorname{supp}(\mathcal{G}) \mid \frac{q}{c}} \lambda(\mathcal{G}) \sum_{h \in s \mathcal{C} \cap L(\mathcal{G})} a(h, c) \Delta(h, c)
$$


Proof. We have that

$$
R_{r}(\mathcal{C}, q)=\frac{1}{N} \sum_{h \in s \mathcal{C} \cap \mathbf{Z}^{r-1}} N(h, q)
$$

By the Chinese Remainder Theorem,

$$
N(h, q)=\prod_{p \mid q} N(h, p)
$$

We rewrite formula (4.3) in the form

$$
N(h, p)=\frac{p+a(h, p)}{2^{r}} \Delta(h, p)
$$

where

$$
\Delta(h, p)=2^{r-r_{e f f}(h)}
$$

Thus we find

$$
N(h, q)=\frac{q \Delta(h, q)}{2^{r \omega(q)}} \sum_{c \mid q} \frac{a(h, c)}{c}=\frac{q}{2^{r \omega(q)}} \sum_{c \mid q} \Delta\left(h, \frac{q}{c}\right) \frac{a(h, c) \Delta(h, c)}{c}
$$

Inserting (5.3) we get a formula for $R_{r}(\mathcal{C}, q)$ : Recalling that $N=q / s$,

$$
R_{r}(\mathcal{C}, q)=\frac{s}{2^{r \omega(q)}} \sum_{c \mid q} \frac{1}{c} \sum_{h \in s \mathcal{C}} \Delta\left(h, \frac{q}{c}\right) a(h, c) \Delta(h, c)
$$

Next we use the expression (4.19) for $\Delta(h, p)$ to write $\Delta\left(h, \frac{q}{c}\right)=$ $\prod_{p \mid q / c} \Delta(h, p)$ in the form

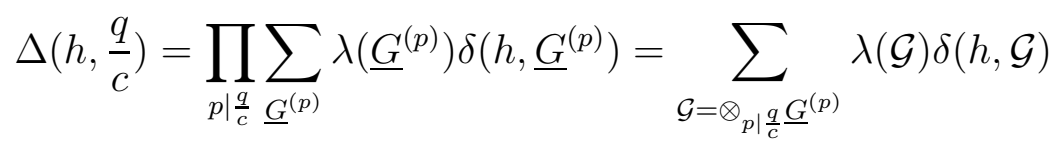

where the sum is over all tuples of set-partitions $\mathcal{G}=\otimes_{p \mid}{ }_{c} \underline{G}^{(p)}$, one for each prime dividing $\frac{q}{c}$, and we put for each such tuple $\mathcal{G}$

$$
\lambda(\mathcal{G}):=\prod_{p \mid \frac{q}{c}} \lambda\left(\underline{G}^{(p)}\right)
$$

and

$$
\delta(h, \mathcal{G}):=\prod_{p \mid \frac{q}{c}} \delta\left(h, \underline{G}^{(p)}\right)= \begin{cases}1 & h \in H_{\underline{G}^{(p)}} \\ 0 & \text { otherwise }\end{cases}
$$


THE DISTRIBUTION OF SPACINGS BETWEEN QUADRATIC RESIDUES 17

This is the characteristic function of the lattice $L(\mathcal{G})$ whose support $\operatorname{supp}(\mathcal{G})$ divides $q / c$. Thus we get the desired expression for $R_{r}(\mathcal{C}, q)$

$$
R_{r}(\mathcal{C}, q)=\frac{s}{2^{r \omega(q)}} \sum_{c \mid q} \frac{1}{c} \sum_{\operatorname{supp}(\mathcal{G}) \mid \frac{q}{c}} \lambda(\mathcal{G}) \sum_{h \in s \mathcal{C} \cap L(\mathcal{G})} a(h, c) \Delta(h, c)
$$




\section{Evaluating the $r$-Level CORRElations}

In order to estimate the correlations using Proposition 6, we partition the sum (5.2) into two parts: the first consisting of pairs $c$ and $\mathcal{G}$ such that $c \operatorname{disc}(\mathcal{G})<s$, and the second of the pairs for which $c \operatorname{disc}(\mathcal{G})>s$. We will show that the first part gives the main term and the second is negligible.

6.1. The case $c \operatorname{disc}(\mathcal{G})>s$. We use $a(h, c) \ll c^{1 / 2+\epsilon}$ (5.1), and $\Delta(h, c) \ll c^{\epsilon}$ to see that this term is bounded by

$$
\frac{s}{2^{r \omega(q)}} \sum_{c \mid q} \frac{1}{c} \sum_{\substack{\operatorname{supp}(\mathcal{G}) \mid \frac{q}{c} \\ c \operatorname{disc}(\mathcal{G})>s}}|\lambda(\mathcal{G})| \#\{s \mathcal{C} \cap L(\mathcal{G})\} c^{1 / 2+\epsilon}
$$

By the Lipschitz principle (Lemma 16),

$$
\#\{s \mathcal{C} \cap L(\mathcal{G})\} \ll \frac{\operatorname{vol}(s \mathcal{C})}{\operatorname{disc}(\mathcal{G})}+s^{r-2}
$$

and since $\operatorname{vol}(s \mathcal{C})=s^{r-1} \operatorname{vol}(\mathcal{C})$, we find that

$$
\#\{s \mathcal{C} \cap L(\mathcal{G})\} \ll \frac{s^{r-1}}{\operatorname{disc}(\mathcal{G})}+s^{r-2}
$$

Moreover, in order that $s \mathcal{C} \cap L(\mathcal{G}) \neq \emptyset$, we will see that we need $\operatorname{supp}(\mathcal{G}) \ll s^{r(r-1) / 2}$, since $\mathcal{C}$ does not intersect the walls. This is a consequence of the following observation: Let $\mathcal{C} \subset \mathbf{R}^{r-1}$ be a bounded convex set. Define

$$
\operatorname{diam}_{1}(\mathcal{C})=\max \left\{\sum_{k=1}^{r-1}\left|x_{k}\right|: x \in \mathcal{C}\right\}
$$

Note that $\operatorname{diam}_{1}$ scales linearly: $\operatorname{diam}_{1}(s \mathcal{C})=s \operatorname{diam}_{1}(\mathcal{C})$ for all $s>0$.

Lemma 7. If $\operatorname{supp}(\mathcal{G})>\operatorname{diam}_{1}(s \mathcal{C})^{r(r-1) / 2}$ then $s \mathcal{C} \cap L(\mathcal{G})$ is contained in the walls $\left\{h \in \mathbf{R}^{r-1}: \sigma_{i j}(h)=0\right.$ for some $\left.i<j\right\}$.

Proof. Let $d_{i j}(\mathcal{G})$ be the product of the primes $p$ such that $\sigma_{i j}$ vanishes on $H_{\underline{G}^{(p)}}$, i.e. so that

$$
\sigma_{i j}(x)=0 \quad \bmod p \quad \text { for all } x \in L(\mathcal{G})
$$

Then $d_{i j}(\mathcal{G}) \mid \operatorname{supp}(\mathcal{G})$ and moreover we claim that:

$$
\operatorname{disc}(\mathcal{G}) \mid \prod_{i<j} d_{i j}(\mathcal{G})
$$


It is enough to check this one prime at a time and is equivalent to saying that

$$
\operatorname{codim}\left(H_{\underline{G}^{(p)}}\right) \leq \#\left\{i<j: \sigma_{i j}=0 \text { on } H_{\underline{G}^{(p)}}\right\}
$$

which follows since $H_{\underline{G}^{(p)}}$ is given by vanishing of some of the $\sigma_{i j}$.

Now note that if $\operatorname{supp}(\mathcal{G})>d^{r(r-1) / 2}$ then for some $i<j, d_{i j}(\mathcal{G})>d$ because $\operatorname{supp}(\mathcal{G}) \leq \operatorname{disc}(\mathcal{G}) \leq \prod_{i<j} d_{i j}(\mathcal{G})$ and the last product consists of $r(r-1) / 2$ factors. If we take $d=\operatorname{diam}_{1}(s \mathcal{C})=s \operatorname{diam}_{1}(\mathcal{C})$, then one has $d_{i j}(\mathcal{G})>\operatorname{diam}_{1}(s \mathcal{C})$ for some $i<j$. However $\sigma_{i j}(h)=0$ $\bmod d_{i j}(\mathcal{G})$ and so $\sigma_{i j}(h)=m d_{i j}(\mathcal{G})$ for some integer $m$. If $m=0$ then $h$ lies in a wall. If $m \neq 0$ then being an integer, $|m| \geq 1$ and so

$$
\left|\sigma_{i j}(h)\right| \geq d_{i j}(\mathcal{G})>\operatorname{diam}_{1}(s \mathcal{C})
$$

Since

$$
\sigma_{i j}(h)=\left|\sum_{k=i}^{j-1} h_{k}\right| \leq \sum_{k=i}^{j-1}\left|h_{k}\right| \leq \sum_{k=i}^{r-1}\left|h_{k}\right|
$$

we find that

$$
\sum_{k=i}^{r-1}\left|h_{k}\right|>\operatorname{diam}_{1}(s \mathcal{C})
$$

Thus $h \notin s \mathcal{C}$ by definition of $\operatorname{diam}_{1}(s \mathcal{C})$.

By Lemma [7, together with $|\lambda(\mathcal{G})| \ll \operatorname{supp}(\mathcal{G})^{\epsilon}$, 6.1) is bounded by

$$
\frac{s}{2^{r \omega(q)}} \sum_{c \mid q} c^{-1 / 2+\epsilon} \sum_{\substack{\operatorname{supp}(\mathcal{G}) \mid \frac{q}{c} \\ c \operatorname{disc}(\mathcal{G})>s \\ \operatorname{supp}(\mathcal{G}) \ll s^{r(r-1) / 2}}} \operatorname{supp}(\mathcal{G})^{\epsilon}\left(\frac{s^{r-1}}{\operatorname{disc}(\mathcal{G})}+s^{r-2}\right)
$$

We split the sum into two parts and use $s<2^{\omega(q)}$ to bound (6.3) by the sum of

$$
\frac{1}{s} \sum_{c \mid q} c^{-1 / 2+\epsilon} \sum_{\substack{\operatorname{supp}(\mathcal{G}) \mid \frac{q}{c} \\ c \operatorname{disc}(\mathcal{G})>s}} \operatorname{supp}(\mathcal{G})^{\epsilon} \frac{s}{\operatorname{disc}(\mathcal{G})}
$$

and

$$
\frac{1}{s} \sum_{c \mid q} c^{-1 / 2+\epsilon} \sum_{\substack{\operatorname{supp}(\mathcal{G}) \mid \frac{q}{c} \\ c \operatorname{disc}(\mathcal{G})>s \\ \operatorname{supp}(\mathcal{G}) \ll s^{r(r-1) / 2}}} \operatorname{supp}(\mathcal{G})^{\epsilon}
$$


We begin by noting that the number of $\mathcal{G}$ with $\operatorname{supp}(\mathcal{G})=g$ is $O\left(g^{\epsilon}\right)$, i.e.,

$$
\sum_{\operatorname{supp}(\mathcal{G})=g} 1 \ll g^{\epsilon}
$$

Since we $\operatorname{sum}$ over $\operatorname{supp}(\mathcal{G}) \ll s^{r(r-1) / 2}$ in $(6.5)$, we have $\operatorname{supp}(\mathcal{G})^{\epsilon} \ll$ $s^{\epsilon^{\prime}}$, and thus (6.5) is bounded by

$$
\frac{1}{s} \sum_{c \mid q} c^{-1 / 2+\epsilon} \sum_{\substack{g \mid \frac{q}{c} \\ g \ll s^{r(r-1) / 2}}} g^{\epsilon} \ll s^{-1+\epsilon} \sum_{c \mid q} c^{-1 / 2+\epsilon} \sum_{\substack{g \mid \frac{q}{c} \\ g \ll s^{r(r-1) / 2}}} 1 .
$$

By Lemma 18, the number of divisors of $q / c$ which are less than $s^{r(r-1) / 2}$ is at most $s^{\epsilon}$, so this term is bounded by

$$
s^{-1+\epsilon} \sum_{c \mid q} c^{-1 / 2+\epsilon} \text {. }
$$

Since

$$
\sum_{c \mid q} c^{-1 / 2+\epsilon}=\prod_{p \mid q}\left(1+\frac{1}{p^{1 / 2-\epsilon}}\right) \ll \prod_{p \mid q}(1+1)^{\epsilon^{\prime}} \ll s^{\epsilon^{\prime \prime}}
$$

the contribution of (6.5) is at most $O\left(s^{-1+\epsilon}\right)$.

It now remains to bound (6.4). We first consider the terms for which $c \operatorname{supp}(\mathcal{G})>s$. Now, $\operatorname{disc}(\mathcal{G}) \geq \operatorname{supp}(\mathcal{G})$, so if $c \operatorname{supp}(\mathcal{G})>s$ then certainly $c \operatorname{disc}(\mathcal{G})>s$, and sum of the corresponding terms in (6.4) is bounded by

$$
\begin{gathered}
\frac{1}{s} \sum_{c \mid q} c^{-1 / 2+\epsilon} \sum_{\substack{\operatorname{supp}(\mathcal{G}) \mid \frac{q}{c} \\
c \operatorname{supp}(\mathcal{G})>s}} \operatorname{supp}(\mathcal{G})^{\epsilon} \frac{s}{\operatorname{supp}(\mathcal{G})} \\
=\sum_{c \mid q} c^{-1 / 2+\epsilon} \sum_{\substack{g \mid \frac{q}{c} \\
c g>s}} \frac{1}{g^{1-\epsilon}} \sum_{\operatorname{supp}(\mathcal{G})=g} 1 \ll \sum_{c \mid q} c^{-1 / 2+\epsilon} \sum_{\substack{g \mid q \\
c g>s}} \frac{1}{g^{1-\epsilon}},
\end{gathered}
$$

by (6.6). Changing variable to $d=c g$, which is a divisor of $q$ satisfying $d>s$, this is bounded by

$$
\sum_{\substack{d \mid q \\ d>s}} \sum_{c \mid d} \frac{c^{-1 / 2+\epsilon}}{(d / c)^{1-\epsilon}}=\sum_{\substack{d \mid q \\ d>s}} \frac{1}{d^{1-\epsilon}} \sum_{c \mid d} c^{1 / 2+\epsilon}
$$

Now the sum $\sum_{c \mid d} c^{1 / 2+\epsilon}$ is bounded by $\tau(d) d^{1 / 2+\epsilon} \ll d^{1 / 2+\epsilon^{\prime}}$, so the above is bounded by

$$
\sum_{\substack{d \mid q \\ d>s}} d^{-1 / 2+\epsilon} \ll s^{-1 / 2+\epsilon}
$$


by Lemma 19. This bounds the contribution of $c, \mathcal{G}$ with $c \operatorname{supp}(\mathcal{G})>s$.

If $c \operatorname{disc}(\mathcal{G})>s$ then $\frac{s}{\operatorname{disc}(\mathcal{G})} \leq c$. This, together with 6.6$)$ implies that

$$
\begin{gathered}
\frac{1}{s} \sum_{c \mid q} c^{-1 / 2+\epsilon} \sum_{\substack{\operatorname{supp}(\mathcal{G}) \mid \frac{q}{c} \\
c \operatorname{disc}(\mathcal{G})>s \\
c \operatorname{supp}(\mathcal{G})<s}} \operatorname{supp}(\mathcal{G})^{\epsilon} \frac{s}{\operatorname{disc}(\mathcal{G})} \\
\ll \frac{1}{s} \sum_{c \mid q} c^{1 / 2+\epsilon} \sum_{\substack{g \mid q \\
c g<s}} g^{\epsilon} \ll s^{-1 / 2+\epsilon} \sum_{\substack{c \mid q \\
c<s}} \sum_{\substack{g \mid q \\
g<s}} 1 \\
\ll s^{-1 / 2+\epsilon}\left(\sum_{\substack{c \mid q \\
c<s}} 1\right)^{2} \ll s^{-1 / 2+\epsilon},
\end{gathered}
$$

since $\sum_{\substack{c \mid q \\ c<s}} 1 \ll s^{\epsilon}$ by lemma 19. Consequently (6.4) is $O\left(s^{-1 / 2+\epsilon}\right)$. (Note that we only used $\operatorname{supp}(\mathcal{G}) \ll s^{r(r-1) / 2}$ to bound $(6.5)$ !)

6.2. The case $c \operatorname{disc}(\mathcal{G}) \leq s$. Fix $c \geq 1$ and $\mathcal{G}$ and partition the lattice points in $s \mathcal{C} \cap L(\mathcal{G})$ into two subsets as follows: Fix a reduced fundamental cell (see B.1) $P=P(\mathcal{G})$ for the lattice $L=L(\mathcal{G})$. Then $c P$ is a reduced fundamental cell for the dilated lattice $c L$. We can tile $\mathbf{R}^{r-1}$ by the translates $h_{c}+c P, h_{c} \in c L$.

Definition 6.1. We say that $x \in L \cap s \mathcal{C}$ is $c$-interior if there is some $y \in c L$ so that $x \in y+c P \subseteq s \mathcal{C}$. We say that $x \in L \cap s \mathcal{C}$ is a $c$-boundary point otherwise.

Note that the notion depends on $c$ and on the choice of a fundamental cell $P$ for $L$.

An important fact is that if $\operatorname{dist}(x, \partial(s \mathcal{C})) \gg_{r} c \operatorname{disc}(L)$ then $x$ is $c$-interior. This follows from Lemma 15 since $\operatorname{diam}(c P) \ll_{r} c \operatorname{disc}(L)$.

Lemma 8. Let $P$ be a fundamental cell for the lattice $L \subseteq \mathbf{Z}^{r-1}, c \geq 1$ so that $\operatorname{gcd}(c, \operatorname{disc}(L))=1$. Then for $y \in c L$, the intersection $L \cap(y+$ $c P)$ with $L$ of the translate of the dilated cell $y+c P$ consists of a full set of representatives of $\mathbf{Z}^{r-1} / c \mathbf{Z}^{r-1}$.

Proof. If $P=\left\{\sum_{j=1}^{r-1} x_{j} \vec{\ell}_{j}: 0 \leq x_{j}<1\right\}$ then the $c^{r-1}$ lattice points $y+\sum_{j=1}^{r-1} n_{j} \vec{\ell}_{j}, n_{j}=0,1, \ldots, c-1$ in $L \cap y+c P$ are clearly inequivalent modulo $c L$, and are the only points of $L$ in this intersection. We will show that if $\operatorname{gcd}(c, \operatorname{disc}(L))=1$ then they are inequivalent modulo $c \mathbf{Z}^{r-1}$. To see this, it suffices to show that $L \cap c \mathbf{Z}^{r-1}=c L$. By the 
theorem on elementary divisors, there is a basis $\left\{\vec{e}_{j}\right\}$ of $\mathbf{Z}^{r-1}$ and integers $d_{j} \geq 1$ so that $\left\{d_{j} \vec{e}_{j}\right\}$ is a basis of $L$, and $\operatorname{disc}(L)=\prod_{j=1}^{r-1} d_{j}$. If $x \in L \cap c \mathbf{Z}^{r-1}$ then $x=\sum_{j=1}^{r-1} m_{j} d_{j} \vec{e}_{j} \in L$ and also $x=c \sum_{j=1}^{r-1} n_{j} \vec{e}_{j} \in$ $c \mathbf{Z}^{r-1}$. Comparing coefficients we find

$$
m_{j} d_{j}=c n_{j}, \quad j=1, \ldots, r-1
$$

Now since $d_{j} \mid \operatorname{disc}(L)$ and $\operatorname{gcd}(c, \operatorname{disc}(L))=1$, we have that $\operatorname{gcd}\left(c, d_{j}\right)=$ 1 and so (6.7) shows that $m_{j}=0 \bmod c$ and $x \in c L$.

Lemma 9. a) The number of points $y$ of $c L$ so that $y+c P \subset s \mathcal{C}$ is

$$
\frac{\operatorname{vol}(s \mathcal{C})}{\operatorname{disc}(c L)}+O\left(\left(\frac{s}{c}\right)^{r-2}\right)
$$

b) The number of c-boundary points of $L$ is $\ll c s^{r-2}$.

Proof. If $y=c z \in c L$ then $y+c P \subseteq s \mathcal{C}$ if and only if $z \in L \cap \frac{s}{c} \mathcal{C}$ and $z+P \subseteq \frac{s}{c} \mathcal{C}$. Thus we need to count $N:=\#\left\{z \in L \cap \frac{s}{c} \mathcal{C}: z+P \subseteq\right.$ $\left.\frac{s}{c} \mathcal{C}\right\}$. An upper bound is obtained by a packing argument - since the translates $z+P$ are disjoint and contained in $\frac{s}{c} \mathcal{C}$, we get

$$
N \operatorname{vol}(P) \leq \operatorname{vol}\left(\frac{s}{c} \mathcal{C}\right)
$$

and so

$$
N \leq \frac{\operatorname{vol}\left(\frac{s}{c} \mathcal{C}\right)}{\operatorname{disc}(L)}=\frac{s^{r-1} \operatorname{vol}(\mathcal{C})}{c^{r-1} \operatorname{disc}(L)}
$$

For a lower bound, note that if $z \in L \cap \frac{s}{c} \mathcal{C}$ satisfies $\operatorname{dist}\left(z, \partial\left(\frac{s}{c} \mathcal{C}\right)\right)>$ $\operatorname{diam}(P)$ then $z+P \subseteq \frac{s}{c} \mathcal{C}$. By the Lipschitz principle (Lemma 16) and Lemma 17, the number $\tilde{N}$ of such points is

$$
\tilde{N}=\frac{\operatorname{vol}\left\{x \in \frac{s}{c} \mathcal{C}: \operatorname{dist}\left(x, \partial\left(\frac{s}{c} \mathcal{C}\right)\right) \geq \operatorname{diam}(P)\right\}}{\operatorname{disc}(L)}+O\left(\left(\frac{s}{c}\right)^{r-2}\right)
$$

Further,

$\operatorname{vol}\left\{x \in \frac{s}{c} \mathcal{C}: \operatorname{dist}\left(x, \partial\left(\frac{s}{c} \mathcal{C}\right)\right) \geq \operatorname{diam}(P)\right\}=\operatorname{vol}\left(\frac{s}{c} \mathcal{C}\right)+O\left(\operatorname{diam}(P)\left(\frac{s}{c}\right)^{r-2}\right)$

and so

$\tilde{N}=\frac{\operatorname{vol}\left(\frac{s}{c} \mathcal{C}\right)}{\operatorname{disc}(L)}+O\left(\frac{\operatorname{diam}(P)\left(\frac{s}{c}\right)^{r-2}}{\operatorname{disc}(L)}+\left(\frac{s}{c}\right)^{r-2}\right)=\frac{\operatorname{vol}\left(\frac{s}{c} \mathcal{C}\right)}{\operatorname{disc}(L)}+O\left(\left(\frac{s}{c}\right)^{r-2}\right)$

because $\operatorname{diam}(P) \ll_{r} \operatorname{disc}(L)$.

Since $N \geq \tilde{N}$, together with the upper bound (6.8) we find

$$
N=\frac{\operatorname{vol}\left(\frac{s}{c} \mathcal{C}\right)}{\operatorname{disc}(L)}+O\left(\left(\frac{s}{c}\right)^{r-2}\right)
$$


b) For the number of $c$-boundary points, we subtract the number of $c$-interior points from the total number of points of $L \cap s \mathcal{C}$. The total number of points in $L \cap s \mathcal{C}$ is by the Lipschitz principle (Lemma 16)

$$
L \cap s \mathcal{C}=\frac{\operatorname{vol}(s \mathcal{C})}{\operatorname{disc}(L)}+O\left(s^{r-2}\right)
$$

To count the number of $c$-interior points, we can write each uniquely as $y+p$, with $y$ as in part a and $p \in L \cap c P$. Now $\#(L \cap c P)=c^{r-1}$ (see Lemma 8) and so by part a, the number $c$-interior points is

$$
N c^{r-1}=\frac{\operatorname{vol}(s \mathcal{C})}{\operatorname{disc}(L)}+O\left(c s^{r-2}\right)
$$

Subtracting (6.10) from (6.9) gives part b.

Fix $\mathcal{G}, c \geq 1$ with $c \operatorname{disc}(\mathcal{G}) \leq s$. Note that since $q$ is square-free and $\operatorname{supp}(\mathcal{G}) \mid \frac{q}{c}$, we have $\operatorname{gcd}(c, \operatorname{disc}(\mathcal{G}))=1$. We now estimate the sum

$$
\sum_{h \in L(\mathcal{G}) \cap s \mathcal{C}} a(h, c) \Delta(h, c)
$$

We divide this into two sums, $\Sigma_{\text {int }}$ over the $c$-interior points and $\Sigma_{b d}$ over the $c$-boundary points. We use $a(h, c) \Delta(h, c) \ll c^{1 / 2+\epsilon}$ to bound $\Sigma_{b d}$ by:

$$
\#\{c \text {-boundary points }\} c^{1 / 2+\epsilon} \ll c s^{r-2} c^{1 / 2+\epsilon}=c^{3 / 2+\epsilon} s^{r-2}
$$

The contribution of the $c$-interior points is computed by writing each such $h$ as $h=y+h_{0}$ with $h_{0} \in c P \cap L$ and $y \in c L \cap s \mathcal{C}$. For each $y$ we get all possible $h_{0}$, which run over a full set of representatives of $\mathbf{Z}^{r-1} / c \mathbf{Z}^{r-1}$ since $\operatorname{gcd}(c, \operatorname{disc}(\mathcal{G}))=1$ (Lemma $\left.\mathbf{8}\right)$. Denote the number of such $y$ by $N$; by Lemma 9 part a, $N=\frac{\operatorname{vol}\left(\frac{s}{c} \mathcal{C}\right)}{\operatorname{disc}(L)}+O\left(\left(\frac{s}{c}\right)^{r-2}\right)$. Moreover

$$
a\left(y+h_{0}, c\right) \Delta\left(y+h_{0}, c\right)=a\left(h_{0}, c\right) \Delta\left(h_{0}, c\right)
$$


since $y \in c L(\mathcal{G}) \subset c \mathbf{Z}^{r-1}$. Thus

$$
\begin{aligned}
\Sigma_{\text {int }} & =N \sum_{h_{0} \bmod c} a\left(h_{0}, c\right) \Delta\left(h_{0}, c\right) \\
& =\left(\frac{\operatorname{vol}\left(\frac{s}{c} \mathcal{C}\right)}{\operatorname{disc}(L)}+O\left(\left(\frac{s}{c}\right)^{r-2}\right)\right) \sum_{h_{0} \bmod c} a\left(h_{0}, c\right) \Delta\left(h_{0}, c\right) \\
& =\frac{\operatorname{vol}\left(\frac{s}{c} \mathcal{C}\right)}{\operatorname{disc}(L)} \sum_{h_{0}} a\left(h_{0}, c\right) \Delta\left(h_{0}, c\right)+O\left(\left(\frac{s}{c}\right)^{r-2} c^{r-1} c^{1 / 2+\epsilon}\right) \\
& =\frac{\operatorname{vol}\left(\frac{s}{c} \mathcal{C}\right)}{\operatorname{disc}(L)} \sum_{h_{0}} a\left(h_{0}, c\right) \Delta\left(h_{0}, c\right)+O\left(c^{3 / 2+\epsilon} s^{r-2}\right)
\end{aligned}
$$

Thus the total contribution of the pairs with $c \operatorname{disc}(\mathcal{G}) \leq s$ is

$$
\begin{gathered}
\frac{s}{2^{r \omega(q)}} \sum_{c \mid q} \frac{1}{c} \sum_{\substack{\operatorname{supp}(\mathcal{G}) \mid \frac{q}{c} \\
c \operatorname{disc}(\mathcal{G}) \leq s}} \lambda(\mathcal{G}) \sum_{h \in s \mathcal{C} \cap L(\mathcal{G})} a(h, c) \Delta(h, c) \\
=\frac{s}{2^{r \omega(q)}} \sum_{c \mid q} \frac{1}{c} \sum_{\substack{\operatorname{supp}(\mathcal{G}) \mid \frac{q}{c} \\
c \operatorname{disc}(\mathcal{G}) \leq s}} \lambda(\mathcal{G}) \frac{\operatorname{vol}(s \mathcal{C})}{c^{r-1} \operatorname{disc}(\mathcal{G})} \sum_{h_{0}} a\left(h_{0}, c\right) \Delta\left(h_{0}, c\right) \\
+O\left(\frac{s}{2^{r \omega(q)}} \sum_{c \mid q} \frac{1}{c} \sum_{\substack{\operatorname{supp}(\mathcal{G}) \mid \frac{q}{c} \\
c \operatorname{disc}(\mathcal{G}) \leq s}}|\lambda(\mathcal{G})| c^{3 / 2+\epsilon} s^{r-2}\right)
\end{gathered}
$$

To estimate the error in 6.11 , note that the condition $c \operatorname{disc}(\mathcal{G}) \leq$ $s$ implies $c \operatorname{supp}(\mathcal{G}) \leq s$ since $\operatorname{supp}(\mathcal{G}) \leq \operatorname{disc}(\mathcal{G})$, so for an upper bound we may replace the summation over pairs satisfying the former condition by the sum over pairs satisfying the latter; this gives (noting that $\left.2^{\omega(q)} \geq s\right)$

$$
\begin{aligned}
\frac{s}{2^{r \omega(q)}} \sum_{c \mid q} \frac{1}{c} \sum_{\substack{\operatorname{supp}(\mathcal{G}) \mid \frac{q}{c} \\
c \operatorname{disc}(\mathcal{G}) \leq s}}|\lambda(\mathcal{G})| c^{3 / 2+\epsilon} s^{r-2} & \ll s^{-1+\epsilon} \sum_{c \mid q} c^{1 / 2+\epsilon} \sum_{\substack{\operatorname{supp}(\mathcal{G}) \mid \frac{q}{c} \\
c \operatorname{supp}(\mathcal{G}) \leq s}}|\lambda(\mathcal{G})| \\
& \ll s^{-1+\epsilon} \sum_{c \mid q} c^{1 / 2+\epsilon} \sum_{\substack{g \mid \frac{q}{c} \\
c g \leq s}} \sum_{\substack{c \mid q \\
\operatorname{supp}(\mathcal{G})=g}}|\lambda(\mathcal{G})|
\end{aligned}
$$

Now $|\lambda(\mathcal{G})| \ll \operatorname{supp}(\mathcal{G})^{\epsilon}$ and the number of $\mathcal{G}$ with $\operatorname{supp}(\mathcal{G})=g$ is $O\left(g^{\epsilon}\right)$, which is $O\left(s^{\epsilon}\right)$ since $g \leq c g \leq s$, so that the above is bounded 
by

$$
s^{-1+\epsilon} \sum_{c \mid q} c^{1 / 2+\epsilon} \sum_{\substack{g \mid \frac{q}{c} \\ c g \leq s}} 1
$$

The number of small divisors $g$ of $\frac{q}{c}$ with $g \leq s / c \leq s$ is at most $s^{\epsilon}$, so the above is at most

$$
s^{-1+\epsilon} \sum_{\substack{c \mid q \\ c \leq s}} c^{1 / 2+\epsilon} \ll s^{-1+\epsilon} s^{1 / 2+\epsilon} \#\{c \mid q: c \leq s\} \ll s^{-1 / 2+\epsilon^{\prime}}
$$

which gives that the error term in (6.11) is $O\left(s^{-1 / 2+\epsilon}\right)$.

We now extend the sum of the first term in (6.11) to all the pairs $c, \mathcal{G}$, introducing an error which was bounded in section 6.1 by $O\left(s^{-1 / 2+\epsilon}\right)$. (This is the term (6.4) which was bounded without using the condition $\operatorname{supp}(\mathcal{G}) \ll s^{r(r-1) / 2}$.)

In summary we find that

\section{Proposition 10.}

$$
\begin{gathered}
R_{r}(\mathcal{C}, q)=\frac{s}{2^{r \omega(q)}} \sum_{c \mid q} \frac{1}{c} \sum_{\operatorname{supp}(\mathcal{G}) \mid \frac{q}{c}} \lambda(\mathcal{G}) \frac{\operatorname{vol}(s \mathcal{C})}{c^{r-1} \operatorname{disc}(L)} \sum_{h_{0}} a\left(h_{0}, c\right) \Delta\left(h_{0}, c\right) \\
+O\left(s^{-1 / 2+\epsilon}\right)
\end{gathered}
$$




\section{The MAIn TERM}

We now treat the main term of (6.12). Define

$$
\mathcal{M}=\frac{s}{2^{r \omega(q)}} \sum_{c \mid q} \frac{1}{c} \sum_{\operatorname{supp}(\mathcal{G}) \mid \frac{q}{c}} \lambda(\mathcal{G}) \frac{\operatorname{vol}(s \mathcal{C})}{c^{r-1} \operatorname{disc}(\mathcal{G})} \sum_{h_{0}} a\left(h_{0}, c\right) \Delta\left(h_{0}, c\right)
$$

we will show

$$
\mathcal{M}=\operatorname{vol}(\mathcal{C})
$$

which with (6.12) will prove Theorem 1.

The sum over $h \bmod c$ is multiplicative:

$$
\sum_{h} a(h, c) \Delta(h, c)=\prod_{p \mid c} \sum_{h} a(h, p) \Delta(h, p)
$$

Furthermore, by Lemma 5

$$
\sum_{h} a(h, p) \Delta(h, p)=(p+1)^{r}-p^{r} \sum_{\underline{G}^{(p)}} \lambda\left(\underline{G}^{(p)}\right) p^{-\operatorname{codim} H_{\underline{G}^{(p)}}}
$$

Now note that since $p^{\operatorname{codim} H_{G}}{ }^{(p)}=\operatorname{disc}\left(\underline{G}^{(p)}\right)$ we get

$$
\begin{aligned}
\mathcal{M} & =\frac{s}{2^{r \omega(q)}} \sum_{c \mid q} \frac{1}{c} \sum_{\operatorname{supp}(\mathcal{G}) \mid \frac{q}{c}} \lambda(\mathcal{G}) \frac{s^{r-1} \operatorname{vol}(\mathcal{C})}{c^{r-1} \operatorname{disc}(\mathcal{G})} \prod_{p \mid c}\left((p+1)^{r}-p^{r} \sum_{\underline{G}^{(p)}} \frac{\lambda\left(\underline{G}^{(p)}\right)}{\operatorname{disc}\left(\underline{G}^{(p)}\right)}\right) \\
& =\frac{\operatorname{vol}(\mathcal{C}) s^{r}}{2^{r \omega(q)}} \sum_{c \mid q} \frac{1}{c^{r}} \sum_{\operatorname{supp}(\mathcal{G}) \mid \frac{q}{c}} \frac{\lambda(\mathcal{G})}{\operatorname{disc}(\mathcal{G})} \prod_{p \mid c}\left((p+1)^{r}-p^{r} \sum_{\underline{G}^{(p)}} \frac{\lambda\left(\underline{G}^{(p)}\right)}{\operatorname{disc}\left(\underline{G}^{(p)}\right)}\right)
\end{aligned}
$$

Further,

$$
\sum_{\operatorname{supp}(\mathcal{G}) \mid \frac{q}{c}} \frac{\lambda(\mathcal{G})}{\operatorname{disc}(\mathcal{G})}=\prod_{p \mid \frac{q}{c}} \sum_{\underline{G}^{(p)}} \frac{\lambda\left(\underline{G}^{(p)}\right)}{\operatorname{disc}\left(\underline{G}^{(p)}\right)}
$$

Therefore we find that

$$
\begin{aligned}
\mathcal{M} & =\operatorname{vol}(\mathcal{C}) \frac{1}{\sigma_{-1}(q)^{r}} \sum_{c \mid q} \prod_{p \mid \frac{q}{c}} \sum_{\underline{G}^{(p)}} \frac{\lambda\left(\underline{G}^{(p)}\right)}{\operatorname{disc}\left(\underline{G}^{(p)}\right)} \prod_{p \mid c}\left(\left(1+\frac{1}{p}\right)^{r}-\sum_{\underline{G}^{(p)}} \frac{\lambda\left(\underline{G}^{(p)}\right)}{\operatorname{disc}\left(\underline{G}^{(p)}\right)}\right) \\
& =\operatorname{vol}(\mathcal{C}) \frac{1}{\sigma_{-1}(q)^{r}} \sum_{c \mid q} A\left(\frac{q}{c}\right) B(c)
\end{aligned}
$$


THE DISTRIBUTION OF SPACINGS BETWEEN QUADRATIC RESIDUES 27

Thus $\mathcal{M}$ is a multiple of the Dirichlet convolution of the multiplicative functions $A, B$, with $A(1)=B(1)=1$,

$$
A(p)=\sum_{\underline{G}^{(p)}} \frac{\lambda\left(\underline{G}^{(p)}\right)}{\operatorname{disc}\left(\underline{G}^{(p)}\right)}
$$

and $\left(\right.$ since $\left.(1+1 / p)^{r}=\sigma_{-1}(p)^{r}\right)$

$$
B(p)=\sigma_{-1}(p)^{r}-A(p)
$$

Now we have

$$
(A * B)(q):=\sum_{c \mid q} A\left(\frac{q}{c}\right) B(c)=\prod_{p \mid q}(A(1) B(p)+A(p) B(1))=\prod_{p \mid q} \sigma_{-1}(p)^{r}=\sigma_{-1}(q)^{r}
$$

by (7.1). This finally gives the main term of $R_{r}(\mathcal{C}, q)$ :

$$
\mathcal{M}=\operatorname{vol}(\mathcal{C}) \frac{1}{\sigma_{-1}(q)^{r}}(A * B)(q)=\operatorname{vol}(\mathcal{C}) \frac{1}{\sigma_{-1}(q)^{r}} \sigma_{-1}(q)^{r}=\operatorname{vol}(\mathcal{C})
$$




\section{Appendix A. Recovering the LEVEl Spacing From the CORRELATIONS}

In this appendix, we explain how to recover the various spacing distributions from the correlation functions. This is well-known in the physics literature (e.g. [15]) and is certainly implicit in Hooley's work [8, 9, 10], but we do not know of a good source for it in the mathematical literature. A very detailed treatment of this and more will appear in a forthcoming book by Katz and Sarnak [12].

We begin with $\mathbf{R} / \mathbf{Z}$ which we think of as the circle with unit circumference. We denote by $\{x\}$ the fractional part of $x$ : If $n \leq x<n+1$, $n$ integer, then $\{x\}=x-n$. We set

$$
((x))= \begin{cases}\{x\} & 0 \leq\{x\}<\frac{1}{2} \\ \{x\}-1 & \frac{1}{2} \leq\{x\}<1\end{cases}
$$

We will order the points in $\mathbf{R} / \mathbf{Z}$ counter-clockwise and write $x \succ y$ if the points lie in a segment of length $<1 / 2$ on $\mathbf{R} / \mathbf{Z}$ and $x$ follows $y$. The signed distance on $\mathbf{R} / \mathbf{Z}$ is given by $((x-y))$; thus $-1 / 2 \leq$ $((x-y))<1 / 2$. In terms of the signed distance, $x \succ y$ if and only if $((x-y))>0$.

Given a finite set $S$ of $N$ points on $\mathbf{R} / \mathbf{Z}$, and $k \geq 2$, the $k$-level correlation functions measure clustering properties of the sequence $S \subset$ $\mathbf{R} / \mathbf{Z}$ on a scale of the mean spacing $1 / N$ : For a $k$-tuple of points $x=\left(x_{1}, \ldots, x_{k}\right)$ of $S$, the oriented distance vector is

$$
D(x)=\left(\left(\left(x_{1}-x_{2}\right)\right), \ldots,\left(\left(x_{k-1}-x_{k}\right)\right)\right)
$$

Given a bounded set $\mathcal{C} \subset \mathbf{R}^{k-1}$, we define the $k$-level correlation as

$$
R_{k}(\mathcal{C}, S)=\frac{1}{N} \#\left\{x \in S^{k}: D(x) \in \frac{1}{N} \mathcal{C}\right\}
$$

As an example, let $\Delta^{k-1} \subset \mathbf{R}^{k-1}$ be the standard open simplex

$$
\Delta^{k-1}=\left\{\left(y_{1}, \ldots y_{k-1}\right) \mid y_{i}>0, \sum_{i=1}^{k-1} y_{i}<1\right\}
$$

and for $t>0$ set $\mathcal{C}=t \Delta^{k-1}$. Then if $N>2 t, D(x) \in \frac{1}{N} \mathcal{C}=\frac{t}{N} \Delta^{k-1}$ means that

1. $\left(\left(x_{i}-x_{i+1}\right)\right)>0$, that is $x_{1} \succ x_{2} \succ \cdots \succ x_{k}$;

2. The points all lie in an arc of length at most $t / N$.

As another example, write $k-1=i+j$ and for $t_{1}, t_{2}>0$ set $\mathcal{C}=t_{1} \Delta^{i} \times t_{2} \Delta^{j}$, which we can write as

$\mathcal{C}=\left\{\left(y_{1}, \ldots y_{k}\right): y_{m}>0, y_{1}+y_{2}+\cdots+y_{i}<t_{1}, y_{i+1}+\cdots+y_{i+j}<t_{2}\right\}$ 
Then $D(x) \in \frac{1}{N} \mathcal{C}$ iff $x_{1} \succ x_{2} \succ \cdots \succ x_{k}$ and $x_{1}, \ldots, x_{i+1}$ lie in an arc of length $<t_{1} / N$, and $x_{i+1}, \ldots, x_{i+j+1}=x_{k}$ lie in an arc of length $<t_{2} / N$.

Given any subset $T \subseteq S$ which is contained in a semi-circle, the ordering gives us unique initial and final elements of $T$, and we can write $T=\left\{x_{\text {init }}=x_{1} \prec x_{2} \prec \cdots \prec x_{\text {fin }}\right\}$. We denote by $|T|$ the number of elements of $T$, and by $\operatorname{diam}(T)$ the distance $\operatorname{dist}\left(x_{i n i t}, x_{f i n}\right)$ between the initial and final points of $T$. If $T$ consists of just the initial and final points, we say that $T$ is a consecutive pair. A consecutive $k$-tuple of $S$ is a $k$-tuple of elements $x_{1}=x_{\text {init }} \prec \cdots \prec x_{k}=x_{\text {fin }}$ so that there are no points of $S$ between $x_{j}$ and $x_{j+1}$, for $1 \leq j<k$.

For $x<1 / 2$, let $N_{k}(x)$ be the number of $k$-tuples of diameter smaller than $x$; this is zero if $k \gg 1$. It is clear from the definitions and the discussion above that we can describe these functions in terms of the correlation function of the simplex $x \Delta^{k-1}$ by

$$
R_{k}\left(x \Delta^{k-1}, S\right)=\frac{1}{N} N_{k}\left(\frac{x}{N}\right)
$$

Further, let $g(x)$ be the number of consecutive pairs of diameter less than $x$, that is the number of spacings between consecutive elements of $S$ of length less than $x$. We may express $g$ in terms of an alternating sum of $N_{k}$ 's as follows:

Lemma 11. With $g$ and $N_{k}$ as above, we have for $x<1 / 2$

$$
g(x)=\sum_{k \geq 2}(-1)^{k} N_{k}(x)
$$

Moreover, for all $n \geq 1$, we have the inequalities

$$
\sum_{k=2}^{2 n+1}(-1)^{k} N_{k}(x) \leq g(x) \leq \sum_{k=2}^{2 n}(-1)^{k} N_{k}(x)
$$

Before giving the proof, we will need the following elementary lemma on sums of binomial coefficients.

Lemma 12. Let $m \geq 0$ be an integer. Then $\sum_{i=0}^{m}(-1)^{i}\left(\begin{array}{c}m \\ i\end{array}\right)=0$ unless $m=0$, in which case the sum equals 1 . Moreover,

$$
\sum_{i=0}^{2 n+1}(-1)^{i}\left(\begin{array}{c}
m \\
i
\end{array}\right) \leq \sum_{i=0}^{m}(-1)^{i}\left(\begin{array}{c}
m \\
i
\end{array}\right) \leq \sum_{i=0}^{2 n}(-1)^{i}\left(\begin{array}{c}
m \\
i
\end{array}\right) .
$$

Proof. The first part is just the binomial expansion of $(1-1)^{m}$. As for the second part, if $m \geq 1$ use the identity $\left(\begin{array}{c}m \\ i\end{array}\right)=\left(\begin{array}{c}m-1 \\ i\end{array}\right)+\left(\begin{array}{c}m-1 \\ i-1\end{array}\right)$ to find $\sum_{i=0}^{k}(-1)^{i}\left(\begin{array}{c}m \\ i\end{array}\right)=(-1)^{k}\left(\begin{array}{c}m-1 \\ k\end{array}\right)$ from which the claim follows. 
We can now prove lemma 11:

Proof. For each pair $T=\{a \succ b\}$, of diameter less than $1 / 2$, we associate $X_{T}$, the set of all $i$-tuples $x_{1} \succ \ldots \succ x_{i}$ in $S$ such that $\left(x_{1}, x_{i}\right)=(a, b)$. The set of all tuples of diameter less than $x$ is thus expressed as a disjoint union of the $X_{T}$ 's as $T$ ranges over all pairs of diameter less than $x$. If we let $N_{i}^{T}$ be the number of $i$ tuples in $X_{T}$ then $N_{i}=\sum_{T} N_{i}^{T}$. But $N_{i}^{T}=\left(\begin{array}{c}|T|-2 \\ i\end{array}\right)$, so by lemma 12, $\sum_{i \geq 2}(-1)^{i} N_{i}^{T}$ is zero unless $T$ is a consecutive pair, in which case the alternating sum is one. Summing over all consecutive pairs we get that $g(x)=\sum_{k \geq 2}(-1)^{k} N_{k}(x)$. Lemma 12 also gives that for $n>0$,

$$
\sum_{i=2}^{2 n+1}(-1)^{i} N_{i}^{T} \leq \sum_{i \geq 2}(-1)^{i} N_{i}^{T} \leq \sum_{i=2}^{2 n}(-1)^{i} N_{i}^{T}
$$

Summing over all $T$ we get the second assertion.

A.1. The joint level spacing. An $(i, j)$-tuple of diameter $(x, y)$ is an $\left(i+j\right.$ )-tuple $x_{1} \succ \ldots \succ x_{i} \succ x_{i+1} \succ \ldots \succ x_{i+j}$ (all lying in an arc of length $<1 / 2)$ such that $\operatorname{dist}\left(x_{i}, x_{1}\right)=x$ and $\operatorname{dist}\left(x_{i+j}, x_{i}\right)=y$.

For $i \geq 2, j \geq 1$ and $x+y<1 / 2$ we let $N_{i, j}(x, y)$ be the number of $(i, j)$-tuples of diameter at most $(x, y)$. Let $g(x, y)$ be the number of consecutive triples $x_{1} \succ x_{2} \succ x_{3}$ of diameter smaller than $(x, y)$. Analogously to lemma 11 we have:

Lemma 13. If we let $A_{k}(x, y)=\sum_{i+j=k} N_{i, j}(x, y)$, then

$$
g(x, y)=\sum_{k \geq 3}(-1)^{k+1} A_{k}(x, y) .
$$

Moreover, for $n \geq 0$ we have the inequalities

$$
\sum_{k=3}^{3+2 n+1}(-1)^{k+1} A_{k}(x, y) \leq g(x, y) \leq \sum_{k=3}^{3+2 n}(-1)^{k+1} A_{k}(x, y) .
$$

Proof. For each triple $T=\{a \succ b \succ c\}$ of diameter at most $(x, y)$, let $X_{T}$ be the set of $(i, j)$-tuples $x_{1} \succ \ldots \succ x_{i} \succ x_{i+1} \succ \ldots \succ x_{i+j}$ such that $\left(x_{1}, x_{i}, x_{i+j}\right)=(a, b, c)$, and let $N_{i, j}^{T}$ be the number of $(i, j)$ tuples in $X_{T}$. We may write the set of $(i, j)$-tuples of diameter smaller than $(x, y)$ as a disjoint union of $X_{T}$ 's, as $T$ ranges over all $(2,1)$ tuples with diameter at most $(x, y)$. Given $T$, we may count tuples of type $(i, j)$ in $X_{T}$ as follows: Let $M, N$ be the number of elements of $S$ between $a, b$ and $b, c$ respectively (we allow both $M$ and $N$ to be zero.) Then $N_{i, j}^{T}=\left(\begin{array}{c}M \\ j-2\end{array}\right)\left(\begin{array}{c}N \\ i-1\end{array}\right)$. Moreover, $A_{k}^{T}=\sum_{i+j=k} N_{i, j}^{T}=\left(\begin{array}{c}M+N \\ k-3\end{array}\right)$ since there are $\left(\begin{array}{c}M+N \\ k-3\end{array}\right)$ ways of choosing $k-3$ objects out of $M$ "blue" 
THE DISTRIBUTION OF SPACINGS BETWEEN QUADRATIC RESIDUES 31 and $N$ "red" objects. By lemma 12, we see that $\sum_{k \geq 3}(-1)^{k+1} A_{k}^{T}=$ $\sum_{k \geq 3}(-1)^{k+1}\left(\begin{array}{c}M+N \\ k-3\end{array}\right)$ is zero unless $T$ is a consecutive $(2,1)$-tuple, in which case it is one. Now, lemma 12 together with $A_{k}^{T}=\left(\begin{array}{c}M+N \\ k-3\end{array}\right)$ shows that

$$
\sum_{k=3}^{3+2 n-1}(-1)^{k+1} A_{k}^{T} \leq \sum_{k \geq 3}(-1)^{k+1} A_{k}^{T} \leq \sum_{k=3}^{3+2 n}(-1)^{k+1} A_{k}^{T} .
$$

Summing over all triples $T$ of diameter at most $(x, y)$ we are done.

\section{A.2. Applications to squares $\bmod q$. We let}

$$
S_{q}=\left\{\frac{n}{q}: 0 \leq n \leq q-1, \quad n \text { a square modulo } q\right\} \subset \mathbf{R} / \mathbf{Z}
$$

be the image in $\mathbf{R} / \mathbf{Z}$ of the set of squares in $\mathbf{Z} / q \mathbf{Z}$. The mean spacing between elements of $S_{q}$ is $1 / N_{q}$, where $N_{q}$ is the number of squares modulo $q$. For $x>0, g_{q}\left(\frac{x}{N_{q}}\right)$ is the number of consecutive pairs in $S_{q}$ of diameter at most $x / N_{q}$, that is the number of normalized consecutive spacings of length $<x$, and we set

$$
\tilde{P}(x)=\lim _{q \rightarrow \infty} \frac{1}{N_{q}} g_{q}\left(\frac{x}{N_{q}}\right)
$$

This is the limiting proportion of normalized consecutive spacings in $S_{q}$ of length at most $x$ (this normalization sets the mean spacing to be unity). $\tilde{P}(x)$ is the cumulant of the level spacing distribution $P(s)$ of the introduction. Likewise we set for $x, y>0$,

$$
\tilde{P}(x, y)=\lim _{q \rightarrow \infty} \frac{1}{N_{q}} g_{q}\left(\frac{x}{N_{q}}, \frac{y}{N_{q}}\right)
$$

the cumulant of the joint level spacing distribution.

For a bounded convex set $\mathcal{C} \subset \mathbf{R}^{k-1}$, not intersecting the walls, and $N \gg 1, \frac{1}{N_{q}} \mathcal{C}$ will be contained in the cube $(-1 / 2,1 / 2)^{k-1}$. For $x=\frac{n}{q} \in S_{q}^{k},\left(0 \leq n_{i}<q\right.$ are squares modulo $\left.q\right)$ the oriented distance vector $D(x)$ (see (A.1) will lie in $\frac{1}{N_{q}} \mathcal{C}$ if and only if there is an integer vector $h \in \frac{q}{N_{q}} \mathcal{C} \cap \mathbf{Z}^{k-1}$ so that

$$
x_{i}-x_{i+1}=h_{i} \bmod q, \quad 1 \leq i \leq k-1
$$

Denoting by $N(h, q)$ the number of solutions of the above system in squares $n_{i}$ modulo $q$, we have found that the correlation function $R_{k}(\mathcal{C}, q):=R_{k}\left(\mathcal{C}, S_{q}\right)$ satisfies

$$
R_{k}(\mathcal{C}, q)=\frac{1}{N_{q}} \sum_{h \in s \mathcal{C} \cap \mathbf{Z}^{k-1}} N(h, q)
$$


with $s=q / N_{q}$.

Lemma 14. If $x, y>0$ then

$$
\tilde{P}(x)=1-e^{-x}
$$

and

$$
\tilde{P}(x, y)=\left(1-e^{-x}\right)\left(1-e^{-y}\right)
$$

Proof. As noted above (see (A.2)), we can express the functions $N_{k}(x)$ in terms of the correlation functions associated to the simplex $x \Delta^{k-1}$, whose volume is $\frac{x^{k-1}}{(k-1) !}$ :

$$
R_{k}\left(x \Delta^{k-1} ; q\right)=\frac{1}{N_{q}} N_{k}\left(\frac{x}{N_{q}}\right)
$$

From theorem 1 we know that

$$
\begin{aligned}
R_{k}\left(x \Delta^{k-1} ; q\right) & =x^{k-1} \operatorname{vol}\left(\Delta^{k-1}\right)+O_{k}\left(s^{-1 / 2+\epsilon}\right) \\
& =\frac{x^{k-1}}{(k-1) !}+O_{k}\left(s^{-1 / 2+\epsilon}\right)
\end{aligned}
$$

By lemma 11] we see that for $n>0$,

$$
\sum_{i=1}^{1+2 n+1}(-1)^{i+1} \frac{x^{i}}{i !} \leq \liminf _{q \rightarrow \infty} \frac{g_{q}\left(\frac{x}{N_{q}}\right)}{N_{q}}
$$

and

$$
\limsup _{q \rightarrow \infty} \frac{g_{q}\left(\frac{x}{N_{q}}\right)}{N_{q}} \leq \sum_{i=1}^{1+2 n}(-1)^{i+1} \frac{x^{i}}{i !}
$$

Letting $n \rightarrow \infty$ and noting that the above polynomials are truncations of the Taylor series of $1-e^{-x}$ we are done.

For the second part of the lemma, recall that $N_{i, j}(x, y)$ is the number of ordered $i+j$-tuples of elements of $S_{q}$ such that the first $i$ are contained in an interval of length $x$, and the last $j$ elements lie in an interval of length $y$. Thus, analogously to (A.2), $N_{i, j}(x, y)$ is a scaled version of the $(i+j-1)$-correlation with respect to the convex set $x \Delta^{i-1} \times y \Delta^{j}$ :

$$
\frac{N_{i, j}\left(\frac{x}{N_{q}}, \frac{y}{N_{q}}\right)}{N_{q}}=R_{i+j}\left(x \Delta^{i-1} \times y \Delta^{j} ; q\right) .
$$

By Theorem 回,

$$
R_{i+j}\left(x \Delta^{i-1} \times y \Delta^{j} ; q\right)=\frac{x^{i-1} y^{j}}{(i-1) ! j !}+O_{i, j}\left(s^{-1 / 2+\epsilon}\right)
$$

since

$$
\operatorname{vol}\left(x \Delta^{i-1} \times y \Delta^{j}\right)=\frac{x^{i-1} y^{j}}{(i-1) ! j !}
$$


THE DISTRIBUTION OF SPACINGS BETWEEN QUADRATIC RESIDUES 33

Letting $A_{k}(x, y)=\sum_{i+j=k} N_{i, j}(x, y)$ and using lemma 12, we get

$$
\limsup _{q \rightarrow \infty} \frac{1}{N_{q}} g_{q}\left(\frac{x}{N_{q}}, \frac{y}{N_{q}}\right) \leq \sum_{k=3}^{3+2 n}(-1)^{k+1} \sum_{\substack{i+j=k \\ i>1 \\ j>0}} \frac{x^{i-1} y^{j}}{(i-1) ! j !}
$$

and

$$
\sum_{k=3}^{3+2 n}(-1)^{k+1} \sum_{\substack{i+j=k \\ i>1 \\ j>0}} \frac{x^{i-1} y^{j}}{(i-1) ! j !} \leq \liminf _{q \rightarrow \infty} \frac{1}{N_{q}} g_{q}\left(\frac{x}{N_{q}}, \frac{y}{N_{q}}\right)
$$

Since the above polynomials are truncations of the Taylor series for $\left(1-e^{-x}\right)\left(1-e^{-y}\right)$, we are done. 


\section{Appendix B. Some Geometry of Numbers}

B.1. Given a basis $\vec{\ell}_{1} \ldots, \vec{\ell}_{n}$ of a lattice $L$ in $\mathbf{R}^{n}$, the fundamental cell is the half-open set

$$
P\left(\left\{\vec{\ell}_{i}\right\}\right):=\left\{x_{1} \vec{\ell}_{1}+\cdots+x_{n} \vec{\ell}_{n}: 0 \leq x_{i}<1\right\}
$$

It serves as a fundamental domain for the action of $L$ on $\mathbf{R}^{n}$ by translations. The volume of $P\left(\left\{\vec{\ell}_{i}\right\}\right)$ is the discriminant $\operatorname{disc}(L)$ of the lattice $L$ :

$$
\operatorname{vol}\left(P\left(\left\{\vec{\ell}_{i}\right\}\right)\right)=\left|\operatorname{det}\left(\vec{\ell}_{1}, \ldots, \vec{\ell}_{n}\right)\right|=\operatorname{disc}(L)
$$

B.2. We need the following basic fact (due to Mahler and Weyl) from reduction theory: In any dimension $n \geq 1$, there are constants $0<c_{n}^{\prime}<$ $c_{n}^{\prime \prime}$ so that any lattice $L \subset \mathbf{R}^{n}$ has a basis $\vec{\ell}_{1} \ldots, \vec{\ell}_{n}$ which is reduced in the sense that

$$
c_{n}^{\prime} \leq \frac{\left|\vec{\ell}_{1}\right| \ldots\left|\vec{\ell}_{n}\right|}{\operatorname{disc}(L)} \leq c_{n}^{\prime \prime}
$$

This is a consequence of Minkowski's second theorem on successive minima; see (Cassels [1], Lemma V.8, p. 135) or (Siegel [20], X §6). This basis is not unique in general.

B.3. We define the diameter $\operatorname{diam}(L)$ of the lattice $L$ to be the minimum of the diameters of all fundamental cells for $L$.

Lemma 15. The diameter of an integer lattice $L \subseteq \mathbf{Z}^{n}$ is bounded by the discriminant of $L$ :

$$
\operatorname{diam}(L) \ll_{n} \operatorname{disc}(L)
$$

the implied constant depending only on the dimension $n$.

Proof. It suffices to show that if $P\left(\left\{\vec{\ell}_{i}\right\}\right)$ is the fundamental cell of an integer lattice $L \subseteq \mathbf{Z}^{n}$ with respect to a reduced basis $\left\{\vec{\ell}_{i}\right\}$, then the diameter of $P\left(\left\{\vec{\ell}_{i}\right\}\right)$ is bounded by the discriminant of $L$ :

$$
\operatorname{diam}\left(P\left(\left\{\vec{\ell}_{i}\right\}\right)\right) \ll_{n} \operatorname{disc}(L)
$$

To see this, note that since $L \subseteq \mathbf{Z}^{n}$ is an integer lattice, the length of any non-zero vector in $L$ is at least 1 , and then this implies that a reduced basis has bounded eccentricity:

$$
1 \leq\left|\vec{\ell}_{1}\right| \leq\left|\vec{\ell}_{2}\right| \leq \cdots \leq\left|\vec{\ell}_{n}\right| \leq c_{n}^{\prime \prime} \operatorname{disc}(L)
$$

(assuming we ordered the basis vectors according to their length). Indeed, using (B.1) together with $\left|\vec{\ell}_{i}\right| \geq 1$ we get an upper bound for the longest basis vector $\vec{\ell}_{n}$

$$
\left|\vec{\ell}_{n}\right|=1 \cdot\left|\vec{\ell}_{n}\right| \leq\left|\vec{\ell}_{1}\right| \cdot\left|\vec{\ell}_{2}\right| \cdots \cdot\left|\vec{\ell}_{n}\right| \leq c_{n}^{\prime \prime} \operatorname{disc}(L)
$$


Thus the diameter of the fundamental cell $P\left(\left\{\vec{\ell}_{i}\right\}\right)$ is at most

$$
\sum_{i=1}^{n}\left|\vec{\ell}_{i}\right| \leq n\left|\vec{\ell}_{n}\right| \leq c_{n}^{\prime \prime} \operatorname{disc}(L)
$$

as required.

B.4. It will be useful to note that for integer dilates $c L$ of a lattice $L$, $c \geq 1$, the diameter scales linearly: $\operatorname{diam}(c L)=c \operatorname{diam}(L)$, while the discriminant scales with $c^{n}: \operatorname{disc}(c L)=c^{n} \operatorname{disc}(L)$. Thus to bound the diameter of a dilate of an integer lattice we use

$$
\operatorname{diam}(c L) \ll_{n} c \operatorname{disc}(L)
$$

\section{B.5. The Lipschitz principle.}

Definition B.1. A set $\mathcal{C} \subset \mathbf{R}^{n}$ is of class $m$ if the intersection of every line with $\mathcal{C}$ consists of at most $m$ intervals (including the degenerate case when some of the intervals are points), and if the same is true for the projection of $\mathcal{C}$ on every linear subspace.

Thus for instance a convex set is of class 1 .

We will use the following form of the "Lipschitz principle" from the geometry of numbers to estimate the number of lattice points in a region of $\mathbf{R}^{n}$ :

Lemma 16. Let $L \subset \mathbf{Z}^{n}$ be an integer lattice of discriminant $\operatorname{disc}(L)$, and $\mathcal{C} \subset \mathbf{R}^{n}$ a set of class $m$ (e.g. a convex set). Suppose that $\mathcal{C}$ lies in a ball of radius $R$ around the origin. Then

$$
\#(L \cap \mathcal{C})=\frac{\operatorname{vol}(\mathcal{C})}{\operatorname{disc}(L)}+O\left(R^{n-1}\right)
$$

This follows from the Lipschitz principle for the integer lattice proven by Davenport [5], as adapted by W. Schmidt ([19], Lemma 1).

We will apply the Lipschitz principle to certain subsets of convex sets. For this purpose we will need:

Lemma 17. Let $\mathcal{C} \subset \mathbf{R}^{n}$ be a convex set, $d>0$ and define

$$
\mathcal{C}_{d}:=\{x \in \mathcal{C}: \operatorname{dist}(x, \partial \mathcal{C}) \geq d\}
$$

to be the set of points of $\mathcal{C}$ of distance at least $d$ from the boundary $\partial \mathcal{C}$ of $\mathcal{C}$. Then $\mathcal{C}_{d}$ is convex.

Proof. What we need to show is that for any $x_{1}, x_{2} \in \mathcal{C}_{d}$, and $\lambda \in[0,1]$, the point $x_{3}=x_{1}+\lambda\left(x_{2}-x_{1}\right)$ also lies in $\mathcal{C}_{d}$, that is if $|y| \leq d$ then $x_{3}+y \in \mathcal{C}$. But $x_{3}+y=\left(x_{1}+y\right)+\lambda\left(\left(x_{2}+y\right)-\left(x_{1}+y\right)\right)$, i.e. $x_{3}+y$ lies on a line between $x_{1}+y$ and $x_{2}+y$. These two points lie in $\mathcal{C}$ since $x_{1}, x_{2} \in \mathcal{C}_{d}$. By convexity so does $x_{3}+y$. 


\section{Appendix C. Counting small Divisors}

In the paper, we need to use some estimates for the number of divisors of $q$ that are smaller than a fixed power of the mean spacing $s$. As is well known, the number of all divisors of $q$ is $O\left(q^{\epsilon}\right)$ for all $\epsilon>0$. This is not enough for our purposes, as we need a bound which is $O\left(s^{\epsilon}\right)$. This is provided by the following lemmas:

Lemma 18. Let $q$ be square-free, $s=2^{\omega(q)} / \sigma_{-1}(q)$. Fix $\alpha>0$. Then as $s \rightarrow \infty$

$$
\#\left\{d \mid q: d<s^{\alpha}\right\}=O\left(s^{\epsilon}\right)
$$

for all $\epsilon>0$.

Proof. We start by bounding products of $k$ distinct primes below by $k^{k}$; we may assume that the primes are the first $k$ primes. Then by the Prime Number Theorem,

$$
\log \prod_{i=1}^{k} p_{i}=\sum_{i=1}^{k} \log p_{i} \sim p_{k} \sim k \log k
$$

Exponentiating we see that the product is bounded below by $k^{k}$. Now,

$$
\#\left\{d \mid q: d<s^{\alpha}\right\}=\sum_{j} a_{j},
$$

where $a_{j}=a\left(j, s^{\alpha}, q\right)$ is the number of divisors of $q$ that are smaller than $s^{\alpha}$ and have precisely $j$ prime factors. But if $j>N$, where $N$ is the smallest integer such that $N^{N} \geq s^{\alpha}$, then $a_{j}=0$. Moreover, setting $w=\omega(q)$, we see that $a_{j} \leq\left(\begin{array}{c}w \\ j\end{array}\right)$. Hence

$$
\sum_{\substack{d \mid q \\
d<s^{\alpha}}} 1 \leq \sum_{j \leq N}\left(\begin{array}{c}
w \\
j
\end{array}\right) \leq N\left(\begin{array}{c}
w \\
N
\end{array}\right)
$$

By Stirling's formula, $\left(\begin{array}{l}w \\ N\end{array}\right) \ll \frac{w^{N}}{(N / e)^{N}}$. Thus

$$
\sum_{i} a_{j} \leq N\left(\begin{array}{c}
w \\
N
\end{array}\right) \ll N\left(\frac{w e}{N}\right)^{N} \ll N\left(\frac{N \log (N) e}{\alpha N \log (2)}\right)^{N}
$$

since $N^{N} \geq s^{\alpha} \gg 2^{w \alpha(1-\epsilon)}$ implies that $w \leq \frac{N \log (N)}{\alpha \log (2)}$. Thus

$$
\left\{d \mid q: d<s^{\alpha}\right\} \ll N\left(\frac{\log (N) e}{\alpha \log (2)}\right)^{N} \ll(C \log N)^{N}
$$

But the last term is clearly $O\left(s^{\epsilon}\right)$.

Lemma 19. If $\alpha>0$ then $\sum_{\substack{d \mid q \\ d>s}} d^{-\alpha} \ll s^{-\alpha+\epsilon}$ 
THE DISTRIBUTION OF SPACINGS BETWEEN QUADRATIC RESIDUES 37

Proof. We divide the sum into two parts: One over $s<d<s^{R}$ and the other over $d>s^{R}$ ( $R$ is a parameter chosen later). For the first, we use the fact that there are few (namely $O\left(s^{\epsilon}\right)$ ) divisors $d$ of $q$ with $d<s^{R}$ to bound that contribution by

$$
\sum_{\substack{d \mid q \\ s<d<s^{R}}} d^{-\alpha} \ll \sum_{\substack{d \mid q \\ s<d<s^{R}}} s^{-\alpha} \ll s^{-\alpha+\epsilon}
$$

For the summands with $d>s^{R}$, use $d^{-\alpha}<s^{-R \alpha}$ and $\tau(q)=2^{\omega(q)} \ll$ $s^{1+\epsilon}$ to get

$$
\sum_{\substack{d \mid q \\ d>s^{R}}} d^{-\alpha} \ll s^{-R \alpha} \tau(q) \ll s^{1-R \alpha+\epsilon}
$$

Now choose $R>0$ so that $1-R \alpha<-\alpha$ to conclude the lemma. 


\section{REFERENCES}

1. J. W. S. Cassels An Introduction to the Geometry of Numbers, Springer-Verlag, Berlin 1959.

2. C. Cobeli and A. Zaharescu On the distribution of primitive roots mod $p$, Acta Arith. 83 (1998), 143-153.

3. H. Davenport On the distribution of quadratic residues $(\bmod p)$ Jour. London Math. Soc. 6 (1931), 49-54, ibid. 8 (1933), 46-52.

4. On character sums in finite fields, Acta Math. 71 (1939), 99-121.

5. H. Davenport On a principle of Lipschitz, J. London Math. Soc. 26 (1951), 179-183.

6. W. Feller An Introduction to Probability Theory and its Applications, Volume II, John Wiley and Sons, Inc. 1966.

7. C. Hooley On the difference of consecutive numbers prime to $n$. I, Acta Arith. $8(1962 / 63), 343-347$.

8. On the difference between consecutive numbers prime to $n$. II, Publ. Math. Debrecen 12 (1965), 39-49.

9. On the difference between consecutive numbers prime to $n$. III, Math. Z. 90 (1965), 355-364.

10. On the intervals between consecutive terms of sequences, Proc. Symp. Pure Math. 24 (1973), 129-140.

11. N. Katz Sommes exponentielles (rédigé par G. Laumon), Asterisque 79 (1980).

12. N. Katz and P. Sarnak Random matrices, Frobenius eigenvalues, and monodromy, American Math. Soc. Colloquium Publications 45, 1999.

13. P. Kurlberg The distribution of spacings between quadratic residues, II submitted for publication.

14. J.H. van Lint and R.M. Wilson A Course in Combinatorics, Cambridge University Press 1992.

15. M.L. Mehta Random Matrices, Second Edition, Academic Press 1991.

16. Z. Rudnick and P. Sarnak The pair correlation function of fractional parts of polynomials, Comm. in Math. Physics. 194 (1998), 61-70.

17. Z. Rudnick, P. Sarnak and A. Zaharescu, in preparation.

18. W. M. Schmidt Equations over finite fields: An elementary approach, Lecture Notes in Math 536 (1976) Springer-Verlag, Berlin Heidelberg.

19. W. M. Schmidt Northcott's theorem on heights II. The quadratic case, Acta Arith. 70 (1995), 343-375.

20. C. L. Siegel Lectures on the Geometry of Numbers (rewritten by K. Chandrasekharan), Springer Verlag 1988.

21. A. Weil Sur les Courbes Algébriques et les Variétés qui s'en Déduisent, Hermann, Paris (1948).

Raymond and Beverly Sackler School of Mathematical Sciences, Tel Aviv University, Tel Aviv 69978, IsRael 